\title{
POSITIVE SOLUTIONS OF HIGHER ORDER QUASILINEAR ELLIPTIC EQUATIONS
}

\author{
MARCELO MONTENEGRO
}

Received 25 February 2002

The higher order quasilinear elliptic equation $-\Delta\left(\Delta_{p}(\Delta u)\right)=f(x, u)$ subject to Dirichlet boundary conditions may have unique and regular positive solution. If the domain is a ball, we obtain a priori estimate to the radial solutions via blowup. Extensions to systems and general domains are also presented. The basic ingredients are the maximum principle, Moser iterative scheme, an eigenvalue problem, a priori estimates by rescalings, sub/supersolutions, and Krasnosel'skii fixed point theorem.

\section{Introduction}

We are interested in studying the higher order quasilinear elliptic equation

$$
-\Delta\left(\Delta_{p}(\Delta u)\right)=f(x, u) \quad \text { in } \Omega, \quad u=0 \quad \text { on } \partial \Omega,
$$

where $\Omega \subset \mathbb{R}^{N}, N \geq 2$, is a smooth bounded domain and $\Delta_{p} u=\operatorname{div}\left(|\nabla u|^{p-2} \nabla u\right)$, $p>1$. Throughout the paper, it is useful to split (1.1) as a system of three equations

$$
\begin{aligned}
-\Delta u_{1} & =u_{2}, \\
-\Delta_{p} u_{2} & =u_{3} \quad \text { in } \Omega, \\
-\Delta u_{3} & =f\left(x, u_{1}\right), \\
u_{1}=u_{2} & =u_{3}=0 \quad \text { on } \partial \Omega .
\end{aligned}
$$

There has been some interest in the study of polyharmonic operators, corresponding to $p=2$ here, see $[4,6,7,9,15]$. These references testify the wide range of applications of higher order elliptic operators. A critical exponent problem involving $\Delta\left(|\Delta u|^{p-2} \Delta u\right)$ was studied in [14], see also [11] for an account on these issues involving polyharmonic operators. Systems dealing with quasilinear equations in radial form were treated in $[2,3]$. They used a blowup method to 
obtain a priori estimates and proved the existence of a solution by degree theoretical arguments. We also take advantage of this general strategy. Here we are concerned with the existence, nonexistence, uniqueness, and regularity of positive solutions to $(1.1)$ whenever $p>1$ and $p \neq 2$. Another goal is to treat systems which are, roughly speaking, a perturbation of (1.2). In this introductory part, we give some examples of our main results, technical assumptions for dealing with general situations are left to other sections.

Problem (1.1) has a variational formulation, so that weak solutions correspond to critical points of the functional

$$
I(u)=\frac{1}{p} \int_{\Omega}|\nabla(\Delta u)|^{p} d x-\int_{\Omega} F(x, u) d x
$$

defined in the Sobolev space

$$
\mathscr{E} p(\Omega)=\left\{u \in W^{2, p}(\Omega) \cap W_{0}^{1, p}(\Omega): \Delta u \in W_{0}^{1, p}(\Omega), 1<p<\infty\right\},
$$

where $F(x, s)=\int_{0}^{s} f(x, t) d t$.

In Theorem 2.2, we employ the so-called Moser iterative scheme to (1.2), in order to regularize the weak solutions of (1.1).

The eigenvalue problem

$$
-\Delta\left(\Delta_{p}(\Delta u)\right)=\Lambda \rho(x)|u|^{p-2} u \quad \text { in } \Omega, \quad u=0 \quad \text { on } \partial \Omega
$$

will help to formulate conditions under which solutions of (1.1) appear. There is a first, positive isolated eigenvalue $\Lambda_{1}^{\rho}$ of the weighted problem (1.5), this is the content of Proposition 3.2.

The radial form of problem (1.1) is interesting because it is possible to obtain an a priori bound for solutions by means of a blowup process, the key step is a Pohozaev identity in the whole $\mathbb{R}^{N}$, see Theorem 4.1 . Notice that the radial ground states of

$$
-\Delta\left(\Delta_{p}(\Delta u)\right)=u^{q} \quad \text { in } \mathbb{R}^{N}
$$

may fail to be sufficiently smooth at $x=0$, therefore, it is not possible to apply directly, for instance, the general program of [10]. We proceed by approximation, writing an integral relation in the annulus $A$ defined by $0<R_{1}<|x|<R_{2}$. A solution of (1.6) and some of its derivatives are bounded near 0 and exhibit rapid decay at $\infty$. This fact allows to take the limits $R_{1} \rightarrow 0$ and $R_{2} \rightarrow \infty$, so we obtain

$$
\int_{\mathbb{R}^{N}}\left(\frac{N}{q+1}-\frac{N-3 p}{p}\right) u^{q+1}(|x|) d x=0 .
$$

Therefore, positive radial solutions of (1.6) defined in the whole $\mathbb{R}^{N}$ cease to exist if $N>3 p$ and $p-1<q<p N /(N-3 p)-1$. We use this information to 
obtain the a priori estimate for positive radial solutions of problem (1.1). In fact, it is possible to work with a class of systems of radial equations that includes (1.1), we pursue this approach in Proposition 5.1. We apply Theorem 5.2 due to Krasnosel'skil to obtain a positive radial solution. The following example is a consequence of Theorem 5.3 and illustrates the preceding comments, notice the relation with the spectral problem (1.5).

Example 1.1. Suppose that for $i=1,2,3$ each function $g_{i}:[0, R] \times[0,+\infty) \rightarrow$ $[0,+\infty)$ is continuous and

$$
g_{i}(r, t) \leq a\left(t^{\beta_{i}}+1\right)
$$

for $r \in[0, R], t \geq 0$ and constants $a>0,0<\beta_{1}, \beta_{2}<1,0<\beta_{3}<q, p-1<q<$ $p N /(N-3 p)-1$, and $N>3 p$. We also assume that

$$
\begin{aligned}
a_{1} t+g_{1}(r, t) & \leq\left(a_{1}+\lambda\right) t, \\
a_{2} t+g_{2}(r, t) & \leq\left(a_{2}+\mu\right) t, \\
a_{3} t^{q}+g_{3}(r, t) & \leq a_{3} t^{q}+\gamma t^{p-1}
\end{aligned}
$$

for $r \in[0, R]$ and $0<t \leq \delta$, where $\lambda, \mu, \gamma>0, a_{i}>0$, and $\left(a_{1}+\lambda\right)^{p-1}\left(a_{2}+\mu\right)\left(a_{3}+\right.$ $\gamma)<\Lambda_{1}^{1}$.

The solutions of the system

$$
\begin{aligned}
-\Delta u_{1} & =a_{1} u_{2}+g_{1}\left(r, u_{2}\right), \\
-\Delta_{p} u_{2} & =a_{2} u_{3}+g_{2}\left(r, u_{3}\right) \quad \text { in } B_{R}, \\
-\Delta u_{3} & =a_{3} u_{1}^{q}+g_{3}\left(r, u_{1}\right), \\
u_{1} & =u_{2}=u_{3}=0 \quad \text { on } \partial B_{R}
\end{aligned}
$$

are a priori bounded, and in fact there is a $C^{1}$ positive weak solution.

One of our aims is to extend results obtained for (1.1) to more general systems of the form

$$
\begin{aligned}
-\Delta u_{1} & =f_{1}\left(x, u_{1}, u_{2}, u_{3}\right), \\
-\Delta_{p} u_{2} & =f_{2}\left(x, u_{1}, u_{2}, u_{3}\right) \quad \text { in } \Omega, \\
-\Delta u_{3} & =f_{3}\left(x, u_{1}, u_{2}, u_{3}\right), \\
u_{1} & =u_{2}=u_{3}=0 \quad \text { on } \partial \Omega,
\end{aligned}
$$

which may not have a straightforward variational structure and $\Omega$ is not a ball. For instance, if we replace the ball $B_{R}$ in Example 1.1 by a smooth bounded 
domain $\Omega$, by Lemma 6.1, we see that there is a nonnegative (maybe identically zero) solution to the corresponding problem in $\Omega$. Essentially, the solution comes up by reducing the problem to the verification of the homotopic invariance of degree in cones. For that matter, we obtain a priori estimates by performing a certain scaling that resembles the blowup method used to prove Proposition 5.1.

The third equation of (1.10) behaves like $q>p-1$ for large values of $u_{1}$. A different behavior at infinity is also treated in the present paper, namely for $q \leq p-1$, see Example 1.2 below. Some additional conditions taking into account the monotonicity of the functions $f_{i}$ permit us to truncate the problem between a positive subsolution and a supersolution, and actually obtain a positive solution, see Theorem 6.2. The next example fits in the general hypotheses of Theorem 6.2 and is different, in nature, from the previous one.

Example 1.2. The system

$$
\begin{aligned}
-\Delta u_{1} & =u_{2}^{\alpha}, \\
-\Delta_{p} u_{2} & =u_{3}^{\beta} \quad \text { in } \Omega, \\
-\Delta u_{3} & =u_{1}^{\gamma}, \\
u_{1}=u_{2} & =u_{3}=0 \quad \text { on } \partial \Omega
\end{aligned}
$$

admits a positive solution, provided that $0<\alpha, \beta \leq 1,0<\gamma \leq p-1$, and $\alpha \beta \gamma<$ $p-1$.

A more general situation occurs when the nonlinearities depend on $u_{1}, u_{2}$, and $u_{3}$. The following example is also a consequence of Theorem 6.2.

Example 1.3. The system has a positive solution

$$
\begin{aligned}
-\Delta u_{1} & =a_{11} u_{1}^{\alpha_{11}}+a_{12} u_{2}^{\alpha_{12}}+a_{13} u_{3}^{\alpha_{13}}, \\
-\Delta_{p} u_{2} & =a_{21} u_{1}^{\alpha_{21}}+a_{22} u_{2}^{\alpha_{22}}+a_{23} u_{3}^{\alpha_{23}} \text { in } \Omega, \\
-\Delta u_{3} & =a_{31} u_{1}^{\alpha_{31}}+a_{32} u_{2}^{\alpha_{32}}+a_{33} u_{3}^{\alpha_{33}}, \\
u_{1} & =u_{2}=u_{3}=0 \quad \text { on } \partial \Omega
\end{aligned}
$$

provided that $a_{i j} \geq 0, a_{12}, a_{23}, a_{31}>0,0<\alpha_{11}, \alpha_{33}<1,0<\alpha_{13}<1 /(p-1), 0<$ $\alpha_{21}, \alpha_{22}, \alpha_{32}<p-1,0<\alpha_{12}, \alpha_{23} \leq 1,0<\alpha_{31} \leq p-1$, and $\alpha_{12} \alpha_{23} \alpha_{31}<p-1$.

The next example is an application of Theorem 6.3, the right-hand side nonlinearities have a different behavior from the previous ones. But even in this situation, it is possible to combine the ideas of Lemma 6.1 in order to get a priori estimate in a suitable homotopy path, similarly to Theorem 5.3 . We finalize by applying Theorem 5.2. 
Example 1.4. Let $g_{i}: \Omega \times[0,+\infty) \rightarrow[0,+\infty), i=1,2,3$, be bounded continuous functions such that

$$
\begin{gathered}
\limsup _{t \rightarrow 0^{+}} g_{1}(x, t)<\lambda<\liminf _{t \rightarrow+\infty} g_{1}(x, t), \\
\limsup _{t \rightarrow 0^{+}} g_{2}(x, t)<\mu<\liminf _{t \rightarrow+\infty} g_{2}(x, t), \\
\limsup _{t \rightarrow 0^{+}} g_{3}(x, t)<\gamma<\liminf _{t \rightarrow+\infty} g_{3}(x, t),
\end{gathered}
$$

uniformly for $x \in \Omega$. If $\lambda^{p-1} \mu \gamma=\Lambda_{1}^{\rho}$, then the system

$$
\begin{aligned}
-\Delta u_{1} & =g_{1}\left(x, u_{2}\right) u_{2}, \\
-\Delta_{p} u_{2} & =g_{2}\left(x, u_{3}\right) u_{3} \quad \text { in } \Omega, \\
-\Delta u_{3} & =g_{3}\left(x, u_{1}\right) \rho(x) u_{1}^{p-1}, \\
u_{1}=u_{2} & =u_{3}=0 \quad \text { on } \partial \Omega
\end{aligned}
$$

possesses a positive weak solution.

It follows from Theorem 7.1 that the systems (1.12) and (1.13) have a unique positive weak solution.

\section{Regularity of weak solutions}

The space $\mathscr{E} p(\Omega)$ is normed by $\|u\|_{\mathscr{E} P(\Omega)}=\left(\int_{\Omega}|\nabla(\Delta u)|^{p} d x\right)^{1 / p}$. In what follows, we obtain embeddings which follow from the continuity of the mappings $\Delta: \mathscr{E} p(\Omega) \rightarrow W_{0}^{1, p}(\Omega)$ and $\Delta^{-1}: L^{v}(\Omega) \rightarrow W^{2, v}(\Omega)$ for $1<v<+\infty$ and from the classical Sobolev embeddings $W_{0}^{1, p}(\Omega) \hookrightarrow L^{v}(\Omega)$ and $W^{2, v}(\Omega) \hookrightarrow L^{\tau}(\Omega)$.

Lemma 2.1. (a) The embedding $\mathscr{E} p(\Omega) \hookrightarrow W^{2, v}(\Omega)$ is continuous for $v \in[1, p N /$ $(N-p)]$ if $p<N$, or for $v \in[1,+\infty)$ if $p \geq N$ and is compact for $v \in$ if $3 p<N$, or for $\tau \in[1,+\infty)$ if $3 p \geq N$ and is compact for $\tau \in\left[1, p^{*}\right)$ if $3 p<N$, or for $\tau \in[1,+\infty)$ if $3 p \geq N$, where $p^{*}=p N /(N-3 p)$. But $\mathscr{E} p(\Omega) \hookrightarrow L^{p^{*}}(\Omega)$ is not compact.

The already defined functional $I$ in $(1.3)$ is of class $C^{1}$ if one assumes that

$$
|f(x, t)| \leq c\left(|t|^{q}+1\right),
$$

for some constant $c>0$ and for $0<q \leq p^{*}-1$ if $3 p<N$ and $0<q<+\infty$ if $3 p \geq$ $N$. The derivative of $I$ is given by

$$
I^{\prime}(u) \varphi=\int_{\Omega}|\nabla(\Delta u)|^{p-2} \nabla(\Delta u) \cdot \nabla(\Delta \varphi) d x-\int_{\Omega} f(x, u) \varphi d x .
$$

We employ a variant of Moser iterative scheme to conclude that weak solutions of (1.1) are regular. If $3 p \geq N$, a weak solution of (1.1) belongs to $C^{3}(\bar{\Omega})$ by a simple application of Lemma 2.1 and $L^{p}$ estimates. 
Theorem 2.2. Let $u \in \mathscr{E} p(\Omega)$ be a weak solution of (1.1). If $q<p^{*}-1$ and $3 p<$ $N$, then $u \in C^{3}(\bar{\Omega})$.

Proof. It is convenient to rewrite (1.1) in the system form (1.2). In this way, we denote $u=u_{1}$ and we claim that there are $u_{2} \in W_{0}^{1, p}(\Omega)$ and $u_{3} \in W_{0}^{1, p^{*} /\left(p^{*}-1\right)}(\Omega)$ such that $\left(u_{1}, u_{2}, u_{3}\right)$ is a weak solution of the system (1.2). Indeed, $u_{1} \in \mathscr{E} P(\Omega)$ is a critical point of $I$, then

$$
\int_{\Omega}\left|\nabla\left(\Delta u_{1}\right)\right|^{p-2} \nabla\left(\Delta u_{1}\right) \cdot \nabla(\Delta \psi) d x=\int_{\Omega} f\left(x, u_{1}\right) \psi d x
$$

for every $\psi \in \mathscr{E} P(\Omega)$. Set $u_{2}=-\Delta u_{1} \in W_{0}^{1, p}(\Omega)$. Then

$$
-\int_{\Omega}\left|\nabla u_{2}\right|^{p-2} \nabla u_{2} \cdot \nabla(\Delta \psi) d x=\int_{\Omega} f\left(x, u_{1}\right) \psi d x
$$

for every $\psi \in \mathscr{E} p(\Omega)$. Since $f\left(x, u_{1}\right) \in L^{\left.p^{* /(} p^{*}-1\right)}(\Omega)$, the problem

$$
-\Delta u_{3}=f\left(x, u_{1}\right) \quad \text { in } \Omega, \quad u_{3}=0 \quad \text { on } \partial \Omega
$$

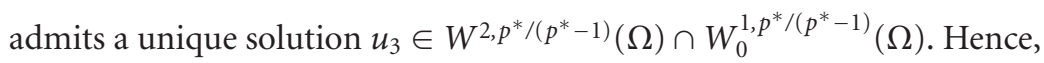

$$
\int_{\Omega} \nabla u_{3} \cdot \nabla \psi d x=\int_{\Omega} f\left(x, u_{1}\right) \psi d x
$$

for every $\psi \in W_{0}^{1, p^{*}}(\Omega)$, implying

$$
-\int_{\Omega} u_{3} \Delta \psi d x=\int_{\Omega} f\left(x, u_{1}\right) \psi d x
$$

for every $\psi \in W^{2, p^{*}}(\Omega) \cap W_{0}^{1, p^{*}}(\Omega)$. From (2.4) and (2.7), we conclude that

$$
\int_{\Omega}\left|\nabla u_{2}\right|^{p-2} \nabla u_{2} \cdot \nabla \varphi d x=\int_{\Omega} u_{3} \varphi d x
$$

for every $\varphi \in C_{0}^{\infty}(\Omega)$. Thus, $\left(u_{1}, u_{2}, u_{3}\right)$ is a weak solution of system (1.2). Now, we prove its regularity. Define the sequence

$$
u_{2 j}(x)= \begin{cases}j & \text { if } u_{2}(x) \geq j \\ u_{2}(x) & \text { if }-j<u_{2}(x)<j \\ -j & \text { if } u_{2}(x) \leq-j\end{cases}
$$


For any given $\beta \geq 0$, we have $\left|u_{2 j}\right|^{\beta} u_{2 j} \in W_{0}^{1, p}(\Omega)$ and

$$
-\int_{\Omega}\left|u_{2 j}\right|^{\beta} u_{2 j} \Delta_{p} u_{2} d x \leq c \int_{\Omega}\left\{(-\Delta)^{-1}\left|(-\Delta)^{-1} u_{2}\right|^{q}+1\right\}\left|u_{2}\right|^{\beta+1} d x .
$$

Suppose that $u_{2} \in L^{p_{k}}(\Omega)$ for some $p_{k} \geq p N /(N-p)$. If $2 p_{k} \geq N$ or $2 p_{k}(q+1) \geq$ $N q$, it is easy to verify that $u_{1} \in L^{\alpha}(\Omega)$ for every $\alpha \in[1,+\infty)$, so we are done. Else, we claim that $u_{2} \in L^{p_{k+1}}(\Omega)$, where

$$
p_{k+1}=\frac{N}{N-p}\left(\beta_{k}+p\right), \quad \beta_{k}=p_{k}-(q+1) \frac{N-2 p_{k}}{N} .
$$

Indeed, since $p_{k} \geq p N /(N-p)$ and $q<p^{*}-1$, it follows that $\beta_{k} \geq 0$. There holds

$$
-\int_{\Omega}\left|u_{2 j}\right|^{\beta_{k}} u_{2 j} \Delta_{p} u_{2} d x \geq\left. c|| u_{2 j}\right|_{L^{p_{k+1}}} ^{\beta_{k}+p}
$$

with $c>0$ independent of $j$, see [8]. Using $L^{p}$ estimates, we obtain

$$
\left\|(-\Delta)^{-1}\left|(-\Delta)^{-1} u_{2}\right|^{q}\right\|_{L^{p_{k} N /\left(\left(N-2 p_{k}\right) q-2 p_{k}\right)}} \leq c\left(\left\|u_{2}\right\|_{L^{p_{k}}}^{q}+1\right) .
$$

Noting that $\left(\beta_{k}+1\right) / p_{k}+\left(\left(N-2 p_{k}\right) q-2 p_{k}\right) / p_{k} N=1$ and applying Young inequality in $(2.10)$, we get

$$
\int_{\Omega}\left\{(-\Delta)^{-1}\left|(-\Delta)^{-1} u_{2}\right|^{q}\right\}\left|u_{2}\right|^{\beta_{k}+1} d x \leq c\left(\|\left. u_{2}\right|_{L^{p_{k}}} ^{q+\beta_{k}+1}+1\right) .
$$

Therefore,

$$
\left\|u_{2 j}\right\|_{L^{p_{k+1}}}^{\beta_{k}+p} \leq c\left(\left\|u_{2}\right\|_{L^{p_{k}}}^{q+\beta_{k}+1}+1\right)
$$

with $c>0$ not depending on $j$. Thus,

$$
\left\|u_{2}\right\|_{L^{p_{k+1}}}^{\beta_{k}+p} \leq \liminf _{j \rightarrow+\infty}\left\|u_{2 j}\right\|_{L^{p_{k+1}}}^{\beta_{k}+p} \leq c\left(\left\|u_{2}\right\|_{L^{p_{k}}}^{q+\beta_{k}+1}+1\right)
$$

proving the claim. Let $p_{0}=p N /(N-p)$, we are going to show that $2 p_{k} \geq N$ or $2 p_{k}(q+1) \geq N q$ for some $k \in \mathbb{N}$. Observe that $p_{k} \geq p_{0}$ for every $k \in \mathbb{N}$ arguing 
by induction, since $p_{k} \geq p_{0}$ implies $\beta_{k} \geq 0$. Note also that, $p_{k}$ is an increasing sequence, by induction and because

$$
p_{k+2}-p_{k+1}=\frac{N+2(q+1)}{N-p}\left(p_{k+1}-p_{k}\right) .
$$

Suppose on the contrary that $2 p_{k}<N$ and $2 p_{k}(q+1)<N q$ for every $k \in \mathbb{N}$. Then $p_{k}$ converges to $L \geq p_{0}$. Using (2.11) and taking the limit

$$
\begin{aligned}
L & =\lim _{k \rightarrow+\infty} p_{k+1}=\frac{N}{N-p} \lim _{k \rightarrow+\infty} \beta_{k}+\frac{p N}{N-p} \\
& =\frac{N}{N-p}\left\{L-\frac{N-2 L}{N}(q+1)\right\}+\frac{p N}{N-p}
\end{aligned}
$$

we see that $L=N(q+1-p) /(p+2(q+1)) \geq p N /(N-p)$, implying that $q+1 \geq$ $p^{*}$, a contradiction.

\section{Eigenvalue problem}

We investigate the eigenvalue problem (1.5). Assume that $\rho$ is a nonnegative and nontrivial function belonging to $L^{\infty}(\Omega)$. Define the functionals $A, B: \mathscr{E} p(\Omega) \rightarrow$ $\mathbb{R}$ by

$$
A(u)=\frac{1}{p}\|u\|_{\mathscr{E} p(\Omega)}^{p}, \quad B(u)=\frac{1}{p} \int_{\Omega} \rho(x)\left(u^{+}\right)^{p} d x,
$$

where $u^{+}=\max \{u, 0\}$. It is easy to verify that $A$ and $B$ are $C^{1}$. Define

$$
\Lambda_{1}^{\rho}=\inf _{B(u)=1} A(u) .
$$

Clearly, $\Lambda_{1}^{\rho}$ is a positive number attained by some $u \in \mathscr{E} P(\Omega)$. Also, there exists $\eta>0$ such that $A^{\prime}(u) \varphi=\eta B^{\prime}(u) \varphi$ for every $\varphi \in \mathscr{E} P(\Omega)$. Taking $\varphi=u$, we obtain $A(u)=\eta B(u)$. Thus, $\eta=\Lambda_{1}^{\rho}$ and $u$ is a critical point of the functional

$$
J(u)=\frac{1}{p} \int_{\Omega}|\nabla(\Delta u)|^{p} d x-\frac{\Lambda_{1}^{\rho}}{p} \int_{\Omega} \rho(x) u^{+p} d x .
$$

The next comparison lemma is borrowed from [12].

Lemma 3.1. Let $u, v \in C^{1}(\bar{\Omega})$ be functions satisfying $-\Delta_{p} u \leq-\Delta_{p} v$ in $\Omega$ and $u \leq$ $v$ on $\partial \Omega$ in the weak sense, then $u \leq v$ in $\Omega$. Furthermore, assume that $\nabla v \neq \equiv$ on $\partial \Omega$ and let $\eta>0$ be small enough, such that the set $\Gamma=\{x \in \Omega:|\nabla v(x)|>$ $\eta$, $\operatorname{dist}(x, \partial \Omega)<\eta\}$ is nonempty and open. Then either $u \equiv v$ in $\Gamma$ or $u<v$ in $\Gamma$ and for each $x \in \partial \Gamma$ with $u(x)=v(x)$, we have $\partial u(x) / \partial v>\partial v(x) / \partial v$.

There is a first eigenvalue associated to problem (1.5), which is isolated from above and from below. 
Proposition 3.2. (i) If $\Lambda=\Lambda_{1}^{\rho}$, then (1.5) admits a positive weak solution;

(ii) if $\Lambda<\Lambda_{1}^{\rho}$, then (1.5) does not admit a positive weak subsolution;

(iii) if $\Lambda>\Lambda_{1}^{\rho}$, then (1.5) does not admit a positive weak supersolution;

(iv) $\Lambda_{1}^{\rho}$ is isolated.

Proof. It is useful to rewrite the eigenvalue problem in the following way:

$$
\begin{aligned}
-\Delta u_{1} & =\lambda u_{2}, \\
-\Delta_{p} u_{2} & =\mu u_{3} \quad \text { in } \Omega, \\
-\Delta u_{3} & =\gamma \rho(x)\left|u_{1}\right|^{p-2} u_{1}, \\
u_{1}=u_{2} & =u_{3}=0 \quad \text { on } \partial \Omega .
\end{aligned}
$$

We reformulate items (i), (ii), and (iii) in terms of a surface in three parameters $\lambda, \mu, \gamma>0$ :

(i) $)^{\prime}$ if $\lambda^{p-1} \mu \gamma=\Lambda_{1}^{\rho}$, then the system (3.4) admits a positive weak solution;

(ii) ${ }^{\prime}$ if $\lambda^{p-1} \mu \gamma<\Lambda_{1}^{\rho}$, then the system (3.4) does not admit a nonnegative weak subsolution with a positive component in $\Omega$;

(iii)' if $\lambda^{p-1} \mu \gamma>\Lambda_{1}^{\rho}$, then the system (3.4) does not admit a positive weak supersolution.

Since $u_{1}$ is a nontrivial critical point of $J$ and $\rho$ is a nonnegative function, (i) ${ }^{\prime}$ follows from the beginning of the proof of Theorem 2.2 and the strong maximum principle of [13]. We prove (ii)' . Suppose on the contrary that problem (3.4) admits a nonnegative weak subsolution $\left(v_{1}, v_{2}, v_{3}\right)$ with a positive component and $\lambda^{p-1} \mu \gamma<\Lambda_{1}^{\rho}$. Choose $\lambda_{0}=\lambda, \mu_{0}=\mu$, and $\gamma_{0}>\gamma$ such that $\lambda_{0}^{p-1} \mu_{0} \gamma_{0}=\Lambda_{1}^{\rho}$. According to part (i)', we can take a positive eigenfunction $\left(u_{1}, u_{2}, u_{3}\right)$ corresponding to $\left(\lambda_{0}, \mu_{0}, \gamma_{0}\right)$. Let $\Gamma_{2}$ be the set associated to function $u_{2}$ given in Lemma 3.1, that is, $\Gamma_{2}=\left\{x \in \Omega:\left|\nabla u_{2}(x)\right|>\eta\right\}$. Define the set $S=\left\{s>0: u_{1}>s v_{1}, u_{2}>s v_{2}\right.$, and $u_{3}>s^{p-1} v_{3}$ in $\left.\Gamma_{2}\right\}$. By the strong maximum principle, $S \neq \varnothing$ and since one of the components of $\left(v_{1}, v_{2}, v_{3}\right)$ is positive, $S$ is bounded. Let $s^{*}=\sup S$. Since

$$
\begin{aligned}
-\Delta\left(u_{3}-s^{* p^{-1}} v_{3}\right) & \geq \gamma_{0} \rho(x) u_{1}^{p-1}-s^{* p-1} \gamma \rho(x) v_{1}^{p-1} \\
& \geq\left(\gamma_{0}-\gamma\right) \rho(x) u_{1}^{p-1} \quad \text { in } \Gamma_{2}
\end{aligned}
$$

by the strong maximum principle, $u_{3}>\left(s^{*}+\varepsilon\right)^{p-1} v_{3}$ in $\Gamma_{2}$ for $\varepsilon>0$ small enough. Thus,

$$
\begin{aligned}
-\Delta_{p} u_{2}+s^{* p-1} \Delta_{p} v_{2} & \geq \mu_{0} u_{3}-s^{* p-1} \mu_{0} v_{3} \\
& \geq \mu_{0}\left\{1-\left(\frac{s^{*}}{s^{*}+\varepsilon}\right)^{p-1}\right\} u_{3} \quad \text { in } \Gamma_{2}
\end{aligned}
$$


implies, by Lemma 3.1, that $u_{2}>\left(s^{*}+\varepsilon\right) v_{2}$ in $\Gamma_{2}$ for $\varepsilon>0$ small enough. Finally, from

$$
-\Delta\left(u_{1}-s^{*} v_{1}\right) \geq \lambda_{0} u_{2}-s^{*} \lambda_{0} v_{2} \geq \lambda_{0}\left(1-\frac{s^{*}}{s^{*}+\varepsilon}\right) u_{2} \quad \text { in } \Gamma_{2},
$$

it follows that $u_{1}>\left(s^{*}+\varepsilon\right) v_{1}$ in $\Gamma_{2}$ for $\varepsilon>0$ is small enough, contradicting the definition of $s^{*}$. Suppose, on the contrary, that problem (3.4) possesses a positive supersolution $\left(v_{1}, v_{2}, v_{3}\right)$. Part (iii)' follows similarly. Let $\lambda_{0}=\lambda, \mu_{0}=\mu$, and $\gamma_{0}<$ $\gamma$ be such that $\lambda_{0}^{p-1} \mu_{0} \gamma_{0}=\Lambda_{1}^{\rho}$. Denote $\left(u_{1}, u_{2}, u_{3}\right)$ a positive eigenfunction related to $\left(\lambda_{0}, \mu_{0}, \gamma_{0}\right)$ and $\Gamma_{2}$ the set associated to $v_{2}$ as in Lemma 3.1. Define the set $S=\left\{s>0: v_{1}>s u_{1}, v_{2}>s u_{2}\right.$, and $v_{3}>s^{p-1} u_{3}$ in $\left.\Gamma_{2}\right\}$. Item (iii) follows by the same steps of (ii) ${ }^{\prime}$. We sketch the proof of item (iv), the details follow from the ideas in [1]. If $v$ is another eigenfunction corresponding to an eigenvalue $\Lambda$, we have $\Lambda \geq \Lambda_{1}^{\rho}$, by (3.2) and (3.3). Hence $\Lambda_{1}^{\rho}$ is isolated to the left. Let $\Lambda_{n}>\Lambda_{1}^{\rho}$ be a sequence of eigenvalues corresponding to the eigenfunctions $v_{n}$. Item (iii) implies that each $v_{n}$ must change sign. The sequence $v_{n}$ converges uniformly in a set of positive measure to the first eigenfunction of (1.5), a contradiction.

\section{Nonexistence of radial solutions in $\mathbb{R}^{N}$}

In this section, we prove a result of Liouville type for (1.6). It is a fundamental step for obtaining a priori estimates in Section 5.

Theorem 4.1. Let $f(t)=|t|^{q-1} t$ with $p-1<q<p^{*}-1$ and $N>3 p$. Then (1.7) has no positive solution in $C^{3}\left(\mathbb{R}^{N}\right)$.

Proof. We rewrite (1.6) as a system of radial equations and proceed by approximation. Suppose that $u$ is a positive solution, (1.6) transforms into

$$
\begin{aligned}
-\left(r^{N-1} u_{1}^{\prime}(r)\right)^{\prime} & =r^{N-1} u_{2}(r), \\
-\left(r^{N-1}\left|u_{2}^{\prime}(r)\right|^{p-2} u_{2}^{\prime}(r)\right)^{\prime} & =r^{N-1} u_{3}(r) \quad \text { for } r>0, \\
-\left(r^{N-1} u_{3}^{\prime}(r)\right)^{\prime} & =r^{N-1} f\left(u_{1}\right),
\end{aligned}
$$

where $u=u_{1}$. The existence of positive $u_{2}$ and $u_{3}$ is treated in Theorem 2.2. A solution $\left(u_{1}, u_{2}, u_{3}\right) \in\left(C^{1}[0,+\infty)\right)^{3}$ of the system $(4$.1) satisfies the integral relations

$$
\begin{aligned}
-r^{N-1} u_{1}^{\prime}(r) & =\int_{0}^{r} s^{N-1} u_{2}(s) d s, \\
-r^{N-1}\left|u_{2}^{\prime}(r)\right|^{p-2} u_{2}^{\prime}(r) & =\int_{0}^{r} s^{N-1} u_{3}(s) d s \quad \text { for } r>0, \\
-r^{N-1} u_{3}^{\prime}(r) & =\int_{0}^{r} s^{N-1} f\left(u_{1}(s)\right) d s,
\end{aligned}
$$


and the following Pohozaev type identity for every constant $a$ and $0<R_{1}<R_{2}$ :

$$
\begin{aligned}
\int_{R_{1}}^{R_{2}}\left\{N F\left(u_{1}(r)\right)-a u_{1}(r) f\left(u_{1}(r)\right)+\left(a+\frac{3 p-N}{p}\right)\left|u_{2}^{\prime}(r)\right|^{p}\right\} r^{N-1} d r \\
\quad=\sum_{i=1}^{2}(-1)^{i} \phi\left(R_{i}, a, u_{1}\left(R_{i}\right), u_{2}\left(R_{i}\right), u_{3}\left(R_{i}\right), u_{1}^{\prime}\left(R_{i}\right), u_{2}^{\prime}\left(R_{i}\right), u_{3}^{\prime}\left(R_{i}\right)\right) R_{i}^{N-1} .
\end{aligned}
$$

We need to detail the expression of $\phi$ in order to verify that $\phi(\cdot) R_{i}^{N-1}$ goes to 0 as $R_{1} \rightarrow 0$ and $R_{2} \rightarrow+\infty$.

Consider the functional $\mathscr{H}=\mathscr{H}\left(x, u_{1}, s\right)$ depending on $x, u_{1}$, and the third derivatives of $u_{1}$ formally represented by $s$,

$$
\mathscr{H}=\frac{1}{p}\left|\nabla\left(\Delta u_{1}(|x|)\right)\right|^{p}-F\left(u_{1}(|x|)\right) \quad \text { for } x \in \mathbb{R}^{N}-\{0\},
$$

where $F$ is the primitive of $f$. By relations (4.2) and a bootstrap argument, we conclude that $\left(u_{1}, u_{2}, u_{3}\right) \in\left(C^{1}[0,+\infty) \cap C^{\infty}(0,+\infty)\right)^{3}$, so $\mathcal{H}$ is well defined. Noting that $s_{i j l}=0$ if $j \neq l$, we obtain

$$
\begin{gathered}
-\int_{A}\left\{N \mathscr{H}-a u_{1} \mathscr{H}_{u_{1}}-(a+3) D_{i j j} u_{1} \mathscr{H}_{s_{i j j}}\right\} d x \\
=\int_{\partial A}\left\{x_{i} \mathscr{H}-\sum_{j, l=1}^{N}\left[\left(x_{l} D_{l} u_{1}+a u_{1}\right) D_{j j} \mathscr{H}_{s_{i j j}}+D_{j}\left(x_{l} D_{l} u_{1}+a u_{1}\right) D_{j} \mathscr{H}_{s_{i j j}}\right.\right. \\
\left.\left.-D_{j j}\left(x_{l} D_{l} u_{1}+a u_{1}\right) \mathscr{H}_{s_{i j j}}\right]\right\} v_{i} d s
\end{gathered}
$$

where $v$ is the unit outward normal vector to the boundary $\partial A$. Since, $u_{1} \mathscr{H}_{u_{1}}=$ $-u_{1} f\left(u_{1}\right)$ and $\sum_{i, j=1}^{N} D_{i j j} u_{1} \mathscr{H}_{s_{i j j}}=\left|\nabla\left(\Delta u_{1}\right)\right|^{p}$, the left-hand side of (4.5) reduces to

$$
\begin{aligned}
\int_{A}\left\{N F\left(u_{1}(r)\right)-a u_{1}(r) f\left(u_{1}(r)\right)+\left(a+\frac{3 p-N}{p}\right)\left|u_{2}^{\prime}(r)\right|^{p}\right\} d x \\
=\omega_{N} \int_{R_{1}}^{R_{2}}\left\{N F\left(u_{1}(r)\right)-a u_{1}(r) f\left(u_{1}(r)\right)+\left(a+\frac{3 p-N}{p}\right)\left|u_{2}^{\prime}(r)\right|^{p}\right\} r^{N-1} d r,
\end{aligned}
$$

where $\omega_{N}$ is the area of the unit $(N-1)$-sphere.

We obtain (4.3) after passing to radial coordinates and replacing $u_{2}$ and $u_{3}$ in (4.5). We also use the fact that $\left(u_{1}, u_{2}, u_{3}\right)$ is a solution of $(4.1)$, translated in the integral relations (4.2). Write each term of the right-hand side integral of (4.5) 
434 Higher order quasilinear elliptic equations

$$
\begin{aligned}
\phi_{1}= & x_{i} \mathcal{H} \frac{x_{i}}{r}=\left[\frac{1}{p}\left|u_{2}^{\prime}(r)\right|^{p}-F\left(u_{1}(r)\right)\right] \frac{x_{i}^{2}}{r}, \\
\phi_{2}= & -\left(x_{l} D_{l} u_{1}+a u_{1}\right)=-u_{1}^{\prime}(r) \frac{x_{l}^{2}}{r}-a u_{1}(r), \\
\phi_{3}= & D_{j j} \mathscr{H}_{s_{i j j}} \frac{x_{i}}{r}=D_{j j}\left(-\left|u_{2}^{\prime}(r)\right|^{p-2} u_{2}^{\prime}(r) \frac{x_{i}}{r}\right) \frac{x_{i}}{r} \\
= & -\left\{D_{j j}\left(\left|u_{2}^{\prime}(r)\right|^{p-2} u_{2}^{\prime}(r)\right) \frac{x_{i}}{r}+2 D_{j}\left(\left|u_{2}^{\prime}(r)\right|^{p-2} u_{2}^{\prime}(r)\right) D_{j} \frac{x_{i}}{r}\right. \\
& \left.+\left|u_{2}^{\prime}(r)\right|^{p-2} u_{2}^{\prime}(r) D_{j j} \frac{x_{i}}{r}\right\} \frac{x_{i}}{r},
\end{aligned}
$$

where

$$
\begin{aligned}
D_{j}\left(\left|u_{2}^{\prime}(r)\right|^{p-2} u_{2}^{\prime}(r)\right)= & \left\{-u_{3}(r)+(N-1) r^{-N} \int_{0}^{r} s^{N-1} u_{3}(s) d s\right\} \frac{x_{j}}{r}, \\
D_{j j}\left(\left|u_{2}^{\prime}(r)\right|^{p-2} u_{2}^{\prime}(r)\right)=\left\{-u_{3}^{\prime}(r) \frac{x_{j}}{r}-N(N-1) r^{-N-1} \frac{x_{j}}{r} \int_{0}^{r} s^{N-1} u_{3}(s) d s\right. & \left.+(N-1) r^{-1} u_{3}(r) \frac{x_{j}}{r}\right\} \frac{x_{j}}{r} \\
& +\left\{u_{3}(r)+(N-1) r^{-N} \int_{0}^{r} s^{N-1} u_{3}(s) d s\right\} D_{j} \frac{x_{j}}{r}, \\
\phi_{4}= & D_{j}\left(x_{l} D_{l} u_{1}+a u_{1}\right)=D_{j}\left(u_{1}^{\prime}(r) r\right)+a D_{j} u_{1}(r) \\
= & -u_{2}(r) x_{j}-(N-2) u_{1}^{\prime}(r) \frac{x_{j}}{r}+a u_{1}^{\prime}(r) \frac{x_{j}}{r}, \\
\phi_{5}= & D_{j} \mathscr{H}_{s_{i j j}} \frac{x_{i}}{r}=D_{j}\left(-\left|u_{2}^{\prime}(r)\right|^{p-2} u_{2}^{\prime}(r) \frac{x_{i}}{r}\right) \frac{x_{i}}{r} \\
= & -\left\{D_{j}\left(\left|u_{2}^{\prime}(r)\right|^{p-2} u_{2}^{\prime}(r)\right) \frac{x_{i}}{r}+\left|u_{2}^{\prime}(r)\right|^{p-2} u_{2}^{\prime}(r) D_{j} \frac{x_{i}}{r}\right\} \frac{x_{i}}{r}, \\
\phi_{6}= & -D_{j j}\left(x_{l} D_{l} u_{1}+a u_{1}\right)=-D_{j j}\left(u_{1}^{\prime}(r) \frac{x_{l}^{2}}{r}\right)-a D_{j j} u_{1}(r),
\end{aligned}
$$

where

$$
\begin{aligned}
D_{j j}\left(u_{1}^{\prime}(r) r\right)= & N(N-2) u_{1}^{\prime}(r) \frac{x_{j}^{2}}{r^{3}}+(N-2) u_{2}(r) \frac{x_{j}^{2}}{r^{2}} \\
& -(N-2) \frac{u_{1}^{\prime}(r)}{r}-u_{2}^{\prime}(r) \frac{x_{j}^{2}}{r}, \\
D_{j j} u_{1}(r)= & -N u_{1}^{\prime}(r) \frac{x_{j}^{2}}{r^{3}}+\frac{u_{1}^{\prime}(r)}{r}-u_{2}(r) \frac{x_{j}^{2}}{r^{2}}, \\
\phi_{7}= & \mathscr{H}_{s_{i j j}} \frac{x_{i}}{r}=-\left|u_{2}^{\prime}(r)\right|^{p-2} u_{2}^{\prime}(r) \frac{x_{i}^{2}}{r^{2}} .
\end{aligned}
$$

Hence $\phi=\phi_{1}+\phi_{2} \phi_{3}+\phi_{4} \phi_{5}+\phi_{6} \phi_{7}$. 
If $\left(u_{1}, u_{2}, u_{3}\right)$ is a positive solution of $(4.1)$, the first member of $(4.3)$ is positive for every $0<R_{1}<R_{2}$. Actually, choosing $a=(N-3 p) / p$, then

$$
N F(t)-a t f(t)=t^{q+1}\left(\frac{N}{q+1}-a\right)>0 \quad \text { for } t>0 .
$$

Now, we intend to prove that the right-hand side of (4.3) converges to zero as $R_{1} \rightarrow 0$ and as $R_{2} \rightarrow+\infty$. We analyze the term $\phi(\cdot) R_{1}^{N-1}$ near zero. Since $u_{i}$ and $u_{i}^{\prime}$ are bounded near 0 for $i=1,2,3$, we get

$$
\begin{aligned}
& \left|\phi_{1}\right| \leq c r \\
& \left|\phi_{2}\right| \leq c(1+r) \\
& \left|\phi_{3}\right| \leq c\left(1+r+r^{-1}+r^{-2}\right), \\
& \left|\phi_{4}\right| \leq c(1+r) \\
& \left|\phi_{5}\right| \leq c\left(1+r+r^{-1}\right), \\
& \left|\phi_{6}\right| \leq c\left(1+r+r^{-1}\right), \\
& \left|\phi_{7}\right| \leq c
\end{aligned}
$$

where the constant $c$ does not depend on $r$ for $r>0$ small enough. Thus, we obtain

$$
\begin{aligned}
& \left|\phi\left(R_{1}, a, u_{1}\left(R_{1}\right), u_{2}\left(R_{1}\right), u_{3}\left(R_{1}\right), u_{1}^{\prime}\left(R_{1}\right), u_{2}^{\prime}\left(R_{1}\right), u_{3}^{\prime}\left(R_{1}\right)\right) R_{1}^{N-1}\right| \\
& \quad \leq c\left(1+R_{1}+R_{1}^{-1}+R_{1}^{-2}\right) R_{1}^{N-1},
\end{aligned}
$$

for every $R_{1}$ near 0 . Since $N \geq 4$, we conclude that $\phi(\cdot) R_{1}^{N-1} \rightarrow 0$ as $R_{1} \rightarrow 0$.

It remains to check the behavior of $\phi(\cdot) R_{2}^{N-1}$ for large $R_{2}$. Here we use a similar strategy to [3]. Integrating by parts the first equation of system (4.1), we have

$$
-r^{N-1} u_{1}^{\prime}(r)=\int_{0}^{r} s^{N-1} u_{2}(s) d s=\left.\frac{s^{N}}{N} u_{2}(s)\right|_{s=0} ^{s=r}-\int_{0}^{r} \frac{s^{N}}{N} u_{2}^{\prime}(s) d s .
$$

Repeating the same computation to the second and third equations of (4.1), we find

$$
-u_{1}^{\prime}(r) \geq \frac{r}{N} u_{2}(r), \quad-u_{2}^{\prime}(r) \geq\left(\frac{r}{N} u_{3}(r)\right)^{1 / p-1}, \quad-u_{3}^{\prime}(r) \geq \frac{r}{N} u_{1}^{q}(r),
$$

for every $r \geq 0$. Therefore,

$$
\begin{aligned}
& -u_{1}^{\prime}(r) \leq(N-2) \frac{u_{1}(r)}{r}, \\
& -u_{2}^{\prime}(r) \leq\left(\frac{N-p}{p-1}\right) \frac{u_{2}(r)}{r}, \\
& -u_{3}^{\prime}(r) \leq(N-2) \frac{u_{3}(r)}{r},
\end{aligned}
$$


for $r>0$. Putting the above relations together, we obtain, step by step, the following estimates for $r>0$ :

$$
\begin{array}{ll}
u_{1}(r) \leq c r^{-3 p /(q+1-p)}, & -u_{1}^{\prime}(r) \leq c r^{-3 p /(q+1-p)-1,}, \\
u_{2}(r) \leq c r^{-3 p /(q+1-p)-2,} & -u_{2}^{\prime}(r) \leq c r^{-3 p /(q+1-p)-3,} \\
u_{3}(r) \leq c r^{-3 p /(q+1-p)-3(p-1)-1,} & -u_{3}^{\prime}(r) \leq c r^{(-3 p /(q+1-p)-3)(p-1)-2},
\end{array}
$$

where the above constant $c$ does not depend on $r$. From (4.16), we see that

$$
\begin{aligned}
& \left|\phi_{1}\right| \leq c\left\{r^{(-3 p /(q+1-p)-3) p}+r^{-3 p(q+1) /(q+1-p)}\right\} r \\
& \left|\phi_{2}\right| \leq c r^{-3 p /(q+1-p)} \\
& \left|\phi_{3}\right| \leq c r^{(-3 p /(q+1-p)-3)(p-1)-2} \\
& \left|\phi_{4}\right| \leq c r^{-3 p /(q+1-p)-1} \\
& \left|\phi_{5}\right| \leq c r^{(-3 p /(q+1-p)-3)(p-1)-1} \\
& \left|\phi_{6}\right| \leq c r^{-3 p /(q+1-p)-2} \\
& \left|\phi_{7}\right| \leq c r^{(-3 p /(q+1-p)-3)(p-1)}
\end{aligned}
$$

where the above constant $c$ does not depend on $r$ for sufficiently large values of $r$. Therefore, it follows that

$$
\left|\phi\left(R_{2}, a, u_{1}\left(R_{2}\right), u_{2}\left(R_{2}\right), u_{3}\left(R_{2}\right), u_{1}^{\prime}\left(R_{2}\right), u_{2}^{\prime}\left(R_{2}\right), u_{3}^{\prime}\left(R_{2}\right)\right) R_{2}^{N-1}\right| \leq c R_{2}^{k}
$$

where $k=-3 p^{2} /(q+1-p)+N-3 p<0$, since $p-1<q<p^{*}-1$. Hence, $\phi(\cdot) R_{2}^{N-1} \rightarrow 0$ as $R_{2} \rightarrow+\infty$. Consequently, relation (4.3) becomes

$$
\int_{0}^{+\infty}\left(\frac{N}{q+1}-\frac{N-3 p}{p}\right) u_{1}^{q+1}(r) r^{N-1} d r=0
$$

implying $u_{1} \equiv 0$ in $[0,+\infty)$, a contradiction.

\section{Existence of radial solutions}

We are going to prove the existence of nontrivial radial solutions for (1.1) in balls. Since our approach can be used to handle more general situations, in fact, we deduce the results for a system like (1.11) that includes (1.1), namely

$$
\begin{aligned}
-\Delta u_{1} & =f_{1}\left(r, u_{1}, u_{2}, u_{3}\right), \\
-\Delta_{p} u_{2} & =f_{2}\left(r, u_{1}, u_{2}, u_{3}\right) \quad \text { in } B_{R}, \\
-\Delta u_{3} & =f_{3}\left(r, u_{1}, u_{2}, u_{3}\right), \\
u_{1} & =u_{2}=u_{3}=0 \quad \text { on } \partial B_{R} .
\end{aligned}
$$


Suppose that, for $i=1,2,3$, each function $f_{i}:[0, R] \times[0,+\infty)^{3} \rightarrow[0,+\infty)$ is continuous and

$$
f_{i}\left(r, t_{1}, t_{2}, t_{3}\right)=g_{i}\left(r, t_{1}, t_{2}, t_{3}\right)+\sum_{l=1}^{3} h_{i l}\left(r, t_{1}, t_{2}, t_{3}\right),
$$

where $g_{i}$ and $h_{i l}$ are nonnegative continuous functions verifying

$$
\begin{gathered}
g_{i}\left(r, t_{1}, t_{2}, t_{3}\right) \leq a\left(t_{1}^{\beta_{i 1}}+t_{2}^{\beta_{i 2}}+t_{3}^{\beta_{i 3}}+1\right), \\
\lim _{t_{l} \rightarrow+\infty} \frac{h_{i l}\left(r, t_{1}, t_{2}, t_{3}\right)}{t_{l}^{\alpha_{i l}}}=a_{i l}
\end{gathered}
$$

for some constant $a>0$, uniformly for $r \in[0, R], t_{k} \in[0,+\infty)$ for every $k=1,2,3$ with $k \neq l$ and

$$
\sup \left\{\left|h_{i l}\left(\cdot, t_{1}, t_{2}, t_{3}\right)\right|: t_{k} \geq 0, k=1,2,3, k \neq l, 0<t_{l}<M\right\} \in L^{\infty}(0, R),
$$

for every $M>0$, where

$$
\begin{gathered}
a_{12}, a_{23}, a_{31}>0, \quad a_{11}, a_{13}, a_{21}, a_{22}, a_{32}, a_{33}=0, \\
\alpha_{i l}, \beta_{i l} \geq 0, \quad \alpha_{12}=\alpha_{23}=1, \quad p-1<\alpha_{31}<p^{*}-1, \\
\beta_{1 l} \gamma_{l}<\gamma_{2}, \quad \beta_{2 l} \gamma_{l}<\gamma_{3}, \quad \beta_{3 l} \gamma_{l}<\alpha_{31} \gamma_{1}, \\
\alpha_{1 l} \gamma_{l} \leq \gamma_{2}, \quad \alpha_{2 l} \gamma_{l} \leq \gamma_{3}, \quad \alpha_{3 l} \gamma_{l} \leq \alpha_{31} \gamma_{1}, \\
\gamma_{1}=\frac{3 p}{\alpha_{31}+1-p}, \quad \gamma_{2}=\frac{2 \alpha_{31}+p+2}{\alpha_{31}+1-p}, \quad \gamma_{3}=\frac{3 p \alpha_{31}+2 p-2 \alpha_{31}-2}{\alpha_{31}+1-p} .
\end{gathered}
$$

We proceed to prove that solutions of (5.1) are a priori bounded. Clearly, (1.1) fits in the above setting if one assumes $|f(r, t)| \leq c\left(|t|^{q}+1\right)$ for $p-1<q<$ $p^{*}-1$. In the previous notation $q=\alpha_{31}, g_{i} \equiv 0, h_{12}=t_{2}, h_{23}=t_{3}, h_{31}=f\left(r, t_{3}\right)$, and others $h_{i l}$ are zero.

Proposition 5.1. There is a constant $c>0$ such that $\left\|u_{i}\right\|_{C[0, R]} \leq c$ for every $i=1,2,3$, where $\left(u_{1}, u_{2}, u_{3}\right) \in\left(C^{1}[0, R]\right)^{3}$ is a radial solution of the system (5.1), provided $3 p<N$ and (5.3), (5.4), and (5.5) hold.

Proof. We write (5.1) as a system of ordinary differential equations. A triplet $\left(u_{1}, u_{2}, u_{3}\right) \in\left(C^{1}[0, R]\right)^{3}$ is a radial solution of system (5.1) if and only if it is a radial weak solution in $\left(C^{1}\left(\overline{B_{R}}\right)\right)^{3}$ of the following problem:

$$
\begin{gathered}
-\left(r^{N-1} u_{1}^{\prime}(r)\right)^{\prime}=r^{N-1} f_{1}\left(r, u_{1}, u_{2}, u_{3}\right), \\
-\left(r^{N-1}\left|u_{2}^{\prime}(r)\right|^{p-2} u_{2}^{\prime}(r)\right)^{\prime}=r^{N-1} f_{2}\left(r, u_{1}, u_{2}, u_{3}\right), \\
-\left(r^{N-1} u_{3}^{\prime}(r)\right)^{\prime}=r^{N-1} f_{3}\left(r, u_{1}, u_{2}, u_{3}\right), \\
u_{1}(R)=u_{2}(R)=u_{3}(R)=0,
\end{gathered}
$$


for $r \in(0, R)$. And it satisfies

$$
\begin{aligned}
& u_{1}(r)=\int_{r}^{R} \frac{1}{s^{N-1}} \int_{0}^{s} t^{N-1} f_{1}\left(t, u_{1}(t), u_{2}(t), u_{3}(t)\right) d t d s, \\
& u_{2}(r)=\int_{r}^{R}\left\{\frac{1}{s^{N-1}} \int_{0}^{s} t^{N-1} f_{2}\left(t, u_{1}(t), u_{2}(t), u_{3}(t)\right) d t\right\}^{1 /(p-1)} d s, \\
& u_{3}(r)=\int_{r}^{R} \frac{1}{s^{N-1}} \int_{0}^{s} t^{N-1} f_{3}\left(t, u_{1}(t), u_{2}(t), u_{3}(t)\right) d t d s,
\end{aligned}
$$

for $r \in[0, R]$. Indeed, if $\left(u_{1}, u_{2}, u_{3}\right) \in\left(C^{1}\left(\overline{B_{R}}\right)\right)^{3}$ is a radial weak solution of (5.1), take $\varphi \in C_{0}^{\infty}(0, R)$, then

$$
\begin{gathered}
\int_{0}^{R} r^{N-1} u_{1}^{\prime}(r) \varphi^{\prime}(r) d r=\frac{1}{\omega_{N}} \int_{B_{R}} \nabla u_{1} \cdot \nabla \varphi d x \\
\int_{0}^{R} r^{N-1} f_{1}\left(r, u_{1}, u_{2}, u_{3}\right) \varphi(r) d r=\frac{1}{\omega_{N}} \int_{B_{R}} f_{1}\left(r, u_{1}, u_{2}, u_{3}\right) \varphi d x .
\end{gathered}
$$

Thus,

$$
\int_{0}^{R} r^{N-1} u_{1}^{\prime}(r) \varphi^{\prime}(r) d r=\int_{0}^{R} r^{N-1} f_{1}\left(r, u_{1}, u_{2}, u_{3}\right) \varphi(r) d r
$$

for every $\varphi \in C_{0}^{\infty}(0, R)$, implying $-\left(r^{N-1} u_{1}^{\prime}(r)\right)^{\prime}=r^{N-1} f_{1}\left(r, u_{1}, u_{2}, u_{3}\right)$ for $r \in$ $(0, R)$. Conversely, take $\left(u_{1}, u_{2}, u_{3}\right) \in\left(C^{1}[0, R]\right)^{3}$ a radial solution of (5.1). Integrating the first equation of (5.7), from 0 to $r$, we get

$$
-u_{1}^{\prime}(r)=\frac{1}{r^{N-1}} \int_{0}^{r} s^{N-1} f_{1}\left(s, u_{1}, u_{2}, u_{3}\right) d s \quad \text { for } r>0 .
$$

Multiplying the above identity by $x \cdot \nabla \varphi(x) / r$ with $\varphi \in C_{0}^{\infty}\left(B_{R}\right)$ and integrating by parts on $B_{R}$, we obtain

$$
\begin{aligned}
\int_{B_{R}} \nabla u_{1} \cdot \nabla \varphi d x & =\int_{B_{R}}\left\{\frac{1}{r^{N}} \int_{0}^{r} s^{N-1} f_{1}\left(s, u_{1}, u_{2}, u_{3}\right) d s\right\} x \cdot \nabla \varphi d x \\
& =\int_{B_{R}} \sum_{i} D_{i}\left\{\frac{x_{i}}{r^{N}} \int_{0}^{r} s^{N-1} f_{1}\left(s, u_{1}, u_{2}, u_{3}\right) d s\right\} \varphi d x \\
& =\int_{B_{R}} f_{1}\left(r, u_{1}, u_{2}, u_{3}\right) \varphi d x
\end{aligned}
$$

The equivalence for other equations of system (5.1) is analogous. From now on, we are going to work with system (5.7).

If the a priori estimate does not hold, there exists a sequence $\left(u_{1 k}, u_{2 k}, u_{3 k}\right) \in$ $\left(C^{1}[0, R]\right)^{3}$ of nonnegative radial solutions of system (5.7) satisfying

$$
t_{j k}=\sup _{r \in[0, R]} u_{j k}(r)=u_{j k}(0) \longrightarrow+\infty \quad \text { as } k \longrightarrow+\infty,
$$


for some $j \in\{1,2,3\}$. Consider the sequence $\lambda_{k}$ defined by $\lambda_{k}=t_{1 k}^{1 / \gamma_{1}}+t_{2 k}^{1 / \gamma_{2}}+$ $t_{3 k}^{1 / \gamma_{3}}$. Since $\gamma_{i}>0$ for $i=1,2,3$, we have

$$
\lambda_{k} \longrightarrow+\infty
$$

Define the rescaling $\tilde{r}=\lambda_{k} r$ and $\tilde{u}_{i k}(\tilde{r})=\left(1 / \lambda_{k}^{\gamma_{i}}\right) u_{i k}(r)$. Since $\tilde{u}_{1 k}^{1 / \gamma_{1}}(0)+\tilde{u}_{2 k}^{1 / \gamma_{2}}(0)+$ $\tilde{u}_{3 k}^{1 / \gamma_{3}}(0)=1$, without loss of generality, we may assume that $\tilde{u}_{i k}(0) \rightarrow \tilde{u}_{i}$ for $i=$ $1,2,3$. In particular,

$$
\tilde{u}_{1}^{1 / \gamma_{1}}+\tilde{u}_{2}^{1 / \gamma_{2}}+\tilde{u}_{3}^{1 / \gamma_{3}}=1
$$

In addition, it is easy to see that $\left(\tilde{u}_{1 k}, \tilde{u}_{2 k}, \tilde{u}_{3 k}\right)$ is nonnegative and satisfy

$$
\begin{gathered}
-\left(\tilde{r}^{N-1} \tilde{u}_{1 k}^{\prime}(\tilde{r})\right)^{\prime}=\lambda_{k}^{-\gamma_{2}} \tilde{r}^{N-1} f_{1}\left(\frac{\tilde{r}}{\lambda_{k}}, \lambda_{k}^{\gamma_{1}} \tilde{u}_{1 k}(\tilde{r}), \lambda_{k}^{\gamma_{2}} \tilde{u}_{2 k}(\tilde{r}), \lambda_{k}^{\gamma_{3}} \tilde{u}_{3 k}(\tilde{r})\right), \\
-\left(\tilde{r}^{N-1}\left|\tilde{u}_{2 k}^{\prime}(\tilde{r})\right|^{p-2} \tilde{u}_{2 k}^{\prime}(\tilde{r})\right)^{\prime}=\lambda_{k}^{-\gamma_{3}} \tilde{r}^{N-1} f_{2}\left(\frac{\tilde{r}}{\lambda_{k}}, \lambda_{k}^{\gamma_{1}} \tilde{u}_{1 k}(\tilde{r}), \lambda_{k}^{\gamma_{2}} \tilde{u}_{2 k}(\tilde{r}), \lambda_{k}^{\gamma_{3}} \tilde{u}_{3 k}(\tilde{r})\right), \\
-\left(\tilde{r}^{N-1} \tilde{u}_{3 k}^{\prime}(\tilde{r})\right)^{\prime}=\lambda_{k}^{-\alpha_{31} \gamma_{1}} \tilde{r}^{N-1} f_{3}\left(\frac{\tilde{r}}{\lambda_{k}}, \lambda_{k}^{\gamma_{1}} \tilde{u}_{1 k}(\tilde{r}), \lambda_{k}^{\gamma_{2}} \tilde{u}_{2 k}(\tilde{r}), \lambda_{k}^{\gamma_{3}} \tilde{u}_{3 k}(\tilde{r})\right) .
\end{gathered}
$$

From (5.4), (5.5), and (5.14), it follows that

$$
\lim _{k \rightarrow+\infty} \lambda_{k}^{-\alpha_{i l} \gamma_{l}} h_{i l}\left(\frac{\tilde{r}}{\lambda_{k}}, \lambda_{k}^{\gamma_{1}} \tilde{u}_{1 k}(\tilde{r}), \lambda_{k}^{\gamma_{2}} \tilde{u}_{2 k}(\tilde{r}), \lambda_{k}^{\gamma_{3}} \tilde{u}_{3 k}(\tilde{r})\right)-a_{i l} \tilde{u}_{l k}^{\alpha_{i l}}(\tilde{r})=0 .
$$

Also, from (5.3), we see that

$$
g_{i}\left(\frac{\tilde{r}}{\lambda_{k}}, \lambda_{k}^{\gamma_{1}} \tilde{u}_{1 k}(\tilde{r}), \lambda_{k}^{\gamma_{2}} \tilde{u}_{2 k}(\tilde{r}), \lambda_{k}^{\gamma_{3}} \tilde{u}_{3 k}(\tilde{r})\right) \leq a\left(\lambda_{k}^{\gamma_{1} \beta_{i 1}}+\lambda_{k}^{\gamma_{2} \beta_{i 2}}+\lambda_{k}^{\gamma_{3} \beta_{i 3}}+1\right)
$$

Therefore,

$$
\begin{array}{r}
\lambda_{k}^{-\gamma_{2}} g_{1}\left(\frac{\tilde{r}}{\lambda_{k}}, \lambda_{k}^{\gamma_{1}} \tilde{u}_{1 k}(\tilde{r}), \lambda_{k}^{\gamma_{2}} \tilde{u}_{2 k}(\tilde{r}), \lambda_{k}^{\gamma_{3}} \tilde{u}_{3 k}(\tilde{r})\right) \\
\lambda_{k}^{-\gamma_{3}} g_{2}\left(\frac{\tilde{r}}{\lambda_{k}}, \lambda_{k}^{\gamma_{1}} \tilde{u}_{1 k}(\tilde{r}), \lambda_{k}^{\gamma_{2}} \tilde{u}_{2 k}(\tilde{r}), \lambda_{k}^{\gamma_{3}} \tilde{u}_{3 k}(\tilde{r})\right) \\
\lambda_{k}^{-\alpha_{31} \gamma_{1}} g_{3}\left(\frac{\tilde{r}}{\lambda_{k}}, \lambda_{k}^{\gamma_{1}} \tilde{u}_{1 k}(\tilde{r}), \lambda_{k}^{\gamma_{2}} \tilde{u}_{2 k}(\tilde{r}), \lambda_{k}^{\gamma_{3}} \tilde{u}_{3 k}(\tilde{r})\right)
\end{array}
$$

uniformly for $\tilde{r} \in\left[0, R \lambda_{k}\right]$. Fix a constant $\tilde{R}>0$. For large enough $k \in \mathbb{N}$, we have $\widetilde{R}<R \lambda_{k}$. Hence it is possible to restrict $\tilde{u}_{i k}$ to $[0, \widetilde{R}]$. Furthermore, we get

$$
\left\|\tilde{u}_{i k}\right\|_{C[0, \tilde{R}]} \leq 1
$$

for $i=1,2,3$. We intend to apply Arzela-Ascoli theorem, so we are going to show that each sequence $\left(\tilde{\mathcal{u}}_{i k}\right)$ is equicontinuous in $C[0, \tilde{R}]$. In fact, from (5.16), we 
conclude that

$$
\begin{aligned}
& -\frac{1}{2} \frac{d}{d \tilde{r}} \tilde{u}_{1 k}^{\prime 2}(\tilde{r})-\frac{n-1}{\tilde{r}} \tilde{u}_{1 k}^{\prime 2}(\tilde{r}) \\
& \quad=\lambda_{k}^{-\gamma_{2}} f_{1}\left(\frac{\tilde{r}}{\lambda_{k}}, \lambda_{k}^{\gamma_{1}} \tilde{u}_{1 k}(\tilde{r}), \lambda_{k}^{\gamma_{2}} \tilde{u}_{2 k}(\tilde{r}), \lambda_{k}^{\gamma_{3}} \tilde{u}_{3 k}(\tilde{r})\right) \tilde{u}_{1 k}^{\prime}(\tilde{r}) \\
& -\frac{p-1}{p} \frac{d}{d \tilde{r}}\left|\tilde{u}_{2 k}^{\prime}(\tilde{r})\right|^{p}-\frac{n-1}{\tilde{r}}\left|\tilde{u}_{2 k}^{\prime}(\tilde{r})\right|^{p} \\
& \quad=\lambda_{k}^{-\gamma_{3}} f_{2}\left(\frac{\tilde{r}}{\lambda_{k}}, \lambda_{k}^{\gamma_{1}} \tilde{u}_{1 k}(\tilde{r}), \lambda_{k}^{\gamma_{2}} \tilde{u}_{2 k}(\tilde{r}), \lambda_{k}^{\gamma_{3}} \tilde{u}_{3 k}(\tilde{r})\right) \tilde{u}_{2 k}^{\prime}(\tilde{r}), \\
& -\frac{1}{2} \frac{d}{d \tilde{r}} \tilde{u}_{3 k}^{\prime 2}(\tilde{r})-\frac{n-1}{\tilde{r}} \tilde{u}_{3 k}^{\prime 2}(\tilde{r}) \\
& \quad=\lambda_{k}^{-\alpha_{31} \gamma_{1}} f_{3}\left(\frac{\tilde{r}}{\lambda_{k}}, \lambda_{k}^{\gamma_{1}} \tilde{u}_{1 k}(\tilde{r}), \lambda_{k}^{\gamma_{2}} \tilde{u}_{2 k}(\tilde{r}), \lambda_{k}^{\gamma_{3}} \tilde{u}_{3 k}(\tilde{r})\right) \tilde{u}_{3 k}^{\prime}(\tilde{r}) .
\end{aligned}
$$

By (5.17) and (5.19), there exists a constant $c>0$ such that

$$
\begin{array}{r}
\lambda_{k}^{-\gamma_{2}} f_{1}\left(\frac{\tilde{r}}{\lambda_{k}}, \lambda_{k}^{\gamma_{1}} \tilde{u}_{1 k}(\tilde{r}), \lambda_{k}^{\gamma_{2}} \tilde{u}_{2 k}(\tilde{r}), \lambda_{k}^{\gamma_{3}} \tilde{u}_{3 k}(\tilde{r})\right) \leq c, \\
\lambda_{k}^{-\gamma_{3}} f_{2}\left(\frac{\tilde{r}}{\lambda_{k}}, \lambda_{k}^{\gamma_{1}} \tilde{u}_{1 k}(\tilde{r}), \lambda_{k}^{\gamma_{2}} \tilde{u}_{2 k}(\tilde{r}), \lambda_{k}^{\gamma_{3}} \tilde{u}_{3 k}(\tilde{r})\right) \leq c, \\
\lambda_{k}^{-\alpha_{31} \gamma_{1}} f_{3}\left(\frac{\tilde{r}}{\lambda_{k}}, \lambda_{k}^{\gamma_{1}} \tilde{u}_{1 k}(\tilde{r}), \lambda_{k}^{\gamma_{2}} \tilde{u}_{2 k}(\tilde{r}), \lambda_{k}^{\gamma_{3}} \tilde{u}_{3 k}(\tilde{r})\right) \leq c .
\end{array}
$$

Thus, from (5.21), we obtain

$$
\begin{gathered}
\frac{1}{2} \frac{d}{d \widetilde{r}} \tilde{u}_{1 k}^{\prime 2}(\tilde{r})+c \tilde{u}_{1 k}^{\prime}(\tilde{r}) \leq 0, \\
\frac{p-1}{p} \frac{d}{d \widetilde{r}}\left|\tilde{u}_{2 k}^{\prime}(\tilde{r})\right|^{p}+c \tilde{u}_{2 k}^{\prime}(\tilde{r}) \leq 0, \\
\frac{1}{2} \frac{d}{d \widetilde{r}} \widetilde{u}_{3 k}^{\prime 2}(\tilde{r})+c \tilde{u}_{3 k}^{\prime}(\tilde{r}) \leq 0 .
\end{gathered}
$$

Integrating the above inequalities from 0 to $\tilde{r}$, we get

$$
\begin{gathered}
\frac{1}{2} \tilde{u}_{1 k}^{\prime 2}(\tilde{r})+c \int_{0}^{\tilde{r}} \tilde{u}_{1 k}^{\prime}(t) d t \leq 0, \\
\frac{p-1}{p}\left|\tilde{u}_{2 k}^{\prime}(\tilde{r})\right|^{p}+c \int_{0}^{\tilde{r}} \tilde{u}_{2 k}^{\prime}(t) d t \leq 0, \\
\frac{1}{2} \tilde{u}_{3 k}^{\prime 2}(\tilde{r})+c \int_{0}^{\tilde{r}} \tilde{u}_{3 k}^{\prime}(t) d t \leq 0,
\end{gathered}
$$

implying that $\left|\tilde{u}_{1 k}^{\prime}(\tilde{r})\right| \leq(2 c)^{1 / 2},\left|\tilde{u}_{2 k}^{\prime}(\tilde{r})\right| \leq((p /(p-1)) c)^{1 / p}$, and $\left|\tilde{u}_{3 k}^{\prime}(\tilde{r})\right| \leq$ $(2 c)^{1 / 2}$ for every $\tilde{r} \in[0, \widetilde{R}]$. Hence, $\left(\tilde{u}_{i k}\right)$ is equicontinuous in $C[0, \widetilde{R}]$ for $i=$ $1,2,3$. By Arzela-Ascoli theorem, up to a subsequence, we have $\tilde{u}_{i k} \rightarrow \tilde{u}_{i}$ in $C[0, \widetilde{R}]$. 
In particular, $\tilde{u}_{i} \geq 0$ in $[0, \tilde{R}]$. From $(5.16)$, it follows that

$$
\tilde{u}_{i k}(0)-\tilde{u}_{i k}(\tilde{r})=\int_{0}^{\tilde{r}} f_{i k}(s) d s,
$$

where

$$
\begin{aligned}
& f_{1 k}(s)=\frac{\lambda_{k}^{-\gamma_{2}}}{s^{N-1}} \int_{0}^{s} t^{N-1} f_{1}\left(\frac{t}{\lambda_{k}}, \lambda_{k}^{\gamma_{1}} \tilde{u}_{1 k}(t), \lambda_{k}^{\gamma_{2}} \tilde{u}_{2 k}(t), \lambda_{k}^{\gamma_{3}} \tilde{u}_{3 k}(t)\right) d t, \\
& f_{2 k}(s)=\left\{\frac{\lambda_{k}^{-\gamma_{3}}}{s^{N-1}} \int_{0}^{s} t^{N-1} f_{2}\left(\frac{t}{\lambda_{k}}, \lambda_{k}^{\gamma_{1}} \tilde{u}_{1 k}(t), \lambda_{k}^{\gamma_{2}} \tilde{u}_{2 k}(t), \lambda_{k}^{\gamma_{3}} \tilde{u}_{3 k}(t)\right) d t\right\}^{1 /(p-1)}, \\
& f_{3 k}(s)=\frac{\lambda_{k}^{-\alpha_{31} \gamma_{1}}}{s^{N-1}} \int_{0}^{s} t^{N-1} f_{3}\left(\frac{t}{\lambda_{k}}, \lambda_{k}^{\gamma_{1}} \tilde{u}_{1 k}(t), \lambda_{k}^{\gamma_{2}} \tilde{u}_{2 k}(t), \lambda_{k}^{\gamma_{3}} \tilde{u}_{3 k}(t)\right) d t .
\end{aligned}
$$

Using (5.17) and (5.19), we conclude that

$$
\begin{aligned}
& \int_{0}^{\tilde{r}} f_{1 k}(s) d s \longrightarrow \int_{0}^{\tilde{r}} \frac{1}{s^{N-1}} \int_{0}^{s} t^{N-1} a_{12} \tilde{u}_{2}(t) d t d s, \\
& \int_{0}^{\tilde{r}} f_{2 k}(s) d s \longrightarrow \int_{0}^{\tilde{r}}\left\{\frac{1}{s^{N-1}} \int_{0}^{s} t^{N-1} a_{23} \tilde{u}_{3}(t) d t\right\}^{1 /(p-1)} d s, \\
& \int_{0}^{\tilde{r}} f_{3 k}(s) d s \longrightarrow \int_{0}^{\tilde{r}} \frac{1}{s^{N-1}} \int_{0}^{s} t^{N-1} a_{31} \tilde{u}_{1}^{\alpha_{31}}(t) d t d s .
\end{aligned}
$$

Letting $k \rightarrow+\infty$ in $(5.25)$, we get a nonnegative solution $\left(\tilde{u}_{1}, \tilde{u}_{2}, \tilde{u}_{3}\right) \in\left(C^{1}[0, \widetilde{R}]\right)^{3}$ of system

$$
\begin{gathered}
-\left(\tilde{r}^{N-1} \tilde{u}_{1}^{\prime}(\tilde{r})\right)^{\prime}=a_{12} \tilde{r}^{N-1} \tilde{u}_{2}(\tilde{r}), \\
-\left(\tilde{r}^{N-1}\left|\tilde{u}_{2}^{\prime}(r)\right|^{p-2} \tilde{u}_{2}^{\prime}(\tilde{r})\right)^{\prime}=a_{23} \tilde{r}^{N-1} \tilde{u}_{3}(\tilde{r}) \quad \text { for } \tilde{r} \in(0, \tilde{R}], \\
-\left(\tilde{r}^{N-1} \tilde{u}_{3}^{\prime}(\tilde{r})\right)^{\prime}=a_{31} \tilde{r}^{N-1} \tilde{u}_{1}^{\alpha_{31}}(\tilde{r}) .
\end{gathered}
$$

A diagonal subsequence argument provides a nonnegative solution $\left(\tilde{u}_{1}, \tilde{u}_{2}, \tilde{u}_{3}\right) \in$ $\left(C^{1}[0,+\infty)\right)^{3}$ of $(5.28)$ in $(0,+\infty)$. By Theorem 4.1 , we conclude that $\tilde{u}_{i} \equiv 0$ in $[0,+\infty)$ for $i=1,2,3$, contradicting $(5.15)$.

Theorem 5.2 (Krasnosel'skiǔ). Let $C$ be a cone in a Banach space $X$ and $T: C \rightarrow C$ a continuous compact mapping with $T(0)=0$. Assume that there exist $t_{0}>0$ and $0<r<R$, such that

(i) $u \neq t T u$, for all $u \in C$ such that $\|u\|_{X}=r$, for all $t \in[0,1]$,

(ii) there exists a continuous compact mapping $H: C \times[0,+\infty) \rightarrow C$ satisfying,

(a) $H(u, 0)=T u$, for all $u \in C$ with $\|u\|_{X} \leq R$,

(b) $H(u, t) \neq u$, for all $u \in C$ with $\|u\|_{X} \leq R$, for all $t \geq t_{0}$,

(c) $H(u, t) \neq u$, for all $u \in C$ with $\|u\|_{X}=R$, for all $t \geq 0$. Then, $T$ has a fixed point $u \in C$ such that $r<\|u\|_{X}<R$. 
The following assumptions are satisfied by Example 1.1,

$$
\begin{aligned}
& f_{1}\left(r, t_{1}, t_{2}, t_{3}\right) \leq \lambda t_{2}, \\
& f_{2}\left(r, t_{1}, t_{2}, t_{3}\right) \leq \mu t_{3}, \\
& f_{3}\left(r, t_{1}, t_{2}, t_{3}\right) \leq \gamma \rho(r) t_{1}^{p-1},
\end{aligned}
$$

for every $r \in(0, R)$ and $0<t_{1}, t_{2}, t_{3} \leq \delta$, where $\rho \in L^{\infty}(0, R), \rho \neq \equiv, \rho \geq 0, \lambda, \mu, \gamma>$ 0 , and $\lambda^{p-1} \mu \gamma<\Lambda_{1}^{\rho}$, where $\Lambda_{1}^{\rho}$ is the first eigenvalue of (1.5).

The above general assumptions are related to the existence of nontrivial solutions of (5.1). Note that (5.29) implies that (1.11) possesses the trivial solution. Equation (1.1) is included in the theorem below, in this particular situation, hypothesis (5.29) is reduced to $f(r, t) \leq \gamma \rho(r) t^{p-1}$ for $\gamma<\Lambda_{1}^{\rho}$ with $\lambda=\mu=1$.

THEOREM 5.3. System (1.11) possesses a nontrivial nonnegative weak solution if (5.3), (5.4), (5.5), and (5.29) are fulfilled.

Proof. Consider the space $X=\left\{u=\left(u_{1}, u_{2}, u_{3}\right) \in(C[0, R])^{3}: u_{i}(R)=0\right.$ for $i=$ $1,2,3\}$ endowed with the norm $\|u\|_{X}=\left\|u_{1}\right\|_{C[0, R]}+\left\|u_{2}\right\|_{C[0, R]}+\left\|u_{3}\right\|_{C[0, R]}$. Denote by $C$ the cone of nonnegative functions of $X$. Define the mapping $H$ : $[0,+\infty) \times C \rightarrow C$ by $H(t, u)=v$, where $v=\left(v_{1}, v_{2}, v_{3}\right)$ with

$$
\begin{aligned}
& v_{1}(r)=\int_{r}^{R}\left\{\frac{1}{\xi^{N-1}} \int_{0}^{\xi} s^{N-1}\left[f_{1}\left(s, u_{1}(s), u_{2}(s), u_{3}(s)\right)+t\right] d s\right\} d \xi, \\
& v_{2}(r)=\int_{r}^{R}\left\{\frac{1}{\xi^{N-1}} \int_{0}^{\xi} s^{N-1}\left[f_{2}\left(s, u_{1}(s), u_{2}(s), u_{3}(s)\right)+t\right] d s\right\}^{1 /(p-1)} d \xi \\
& v_{3}(r)=\int_{r}^{R}\left\{\frac{1}{\xi^{N-1}} \int_{0}^{\xi} s^{N-1}\left[f_{3}\left(s, u_{1}(s), u_{2}(s), u_{3}(s)\right)+t\right] d s\right\} d \xi
\end{aligned}
$$

for every $r \in[0, R]$. It is easy to see that the mapping $H$ is well defined, continuous and compact. Let $T: C \rightarrow C$ be given by $T(u)=H(0, u)$. Then $T(0)=0$. Now we seek $r_{0}>0$ such that $u \neq t T(u)$ for every $t \in[0,1]$ and $u \in C$ with $\|u\|_{X}=r_{0}$. Take $\delta \geq r_{0}$. If $u=t T(u)$ for some $t \in[0,1]$ and $u \in C$ with $\|u\|_{X}=r_{0}$. Then, from Proposition 5.1 and (5.29), we conclude that $\left(u_{1}, u_{2}, u_{3}\right) \in\left(C^{1}\left(\overline{B_{R}}\right)\right)^{3}$ and

$$
\begin{aligned}
-\Delta u_{1} & =t f_{1}\left(r, u_{1}, u_{2}, u_{3}\right) \leq \lambda u_{2}, \\
-\Delta_{p} u_{2} & =t^{p-1} f_{2}\left(r, u_{1}, u_{2}, u_{3}\right) \leq \mu u_{3} \quad \text { in } B_{R}, \\
-\Delta u_{3} & =t f_{3}\left(r, u_{1}, u_{2}, u_{3}\right) \leq \gamma \rho(r) u_{1}^{p-1}, \\
u_{1} & =u_{2}=u_{3}=0 \quad \text { on } \partial B_{R},
\end{aligned}
$$

in the weak sense. Since $u$ has a positive component in $\Omega$, by Proposition 3.2(ii), we obtain a contradiction. We claim that there exist $R>r_{0}$ and $t_{0}>0$ such that $H(t, u) \neq u$ for every $t \geq t_{0}$ and $u \in C$ with $\|u\|_{X} \leq R$. Also $H(t, u) \neq u$ in $C$ for 
the same $r_{0}$ and for every $t \geq 0$ when $\|u\|_{X}=R$. Indeed, let $t \geq 0$ and $u \in C$ verify $H(t, u)=u$. From (5.4) and (5.5), we get

$$
\begin{aligned}
& f_{1}\left(r, t_{1}, t_{2}, t_{3}\right) \geq \tilde{\lambda} t_{2}-c_{1}, \\
& f_{2}\left(r, t_{1}, t_{2}, t_{3}\right) \geq \tilde{\mu} t_{3}-c_{1}, \\
& f_{3}\left(r, t_{1}, t_{2}, t_{3}\right) \geq \tilde{\gamma} t_{1}^{p-1}-c_{1},
\end{aligned}
$$

for every $r \in[0, R]$ and $t_{1}, t_{2}, t_{3} \geq 0$, where $\tilde{\lambda}, \tilde{\mu}, \tilde{\gamma}>0$ and $\tilde{\lambda}^{p-1} \tilde{\mu} \tilde{\gamma}>\Lambda_{1}^{1}$. Again, by Proposition 5.1 and (5.32), we have

$$
\begin{gathered}
-\Delta u_{1} \geq \tilde{\lambda} u_{2}+t-c_{1}, \\
-\Delta_{p} u_{2} \geq \tilde{\mu} u_{3}+t-c_{1} \quad \text { in } B_{R}, \\
-\Delta u_{3} \geq \tilde{\gamma} u_{1}^{p-1}+t-c_{1}, \\
u_{1}=u_{2}=u_{3}=0 \quad \text { on } \partial B_{R},
\end{gathered}
$$

in the weak sense. Applying Proposition 3.2(iii), we obtain $t \leq c_{1}$. By Proposition 5.1 , we conclude that $\|u\|_{X} \leq c$. It is enough to take $R>c$ and $t_{0}>c_{1}$. The conclusion follows from Krasnosel'skiĭ theorem (Theorem 5.2).

\section{Further generalizations to nonradial systems}

Some classes of general systems (1.11) possess positive solutions. The next lemma is a fundamental preliminary result in this direction and can be viewed as an extension of Theorem 5.3 to more general domains. We deduce an a priori estimate and the existence of a nonnegative solution by using the homotopic invariance of degree in cones.

Assume that

$$
\begin{aligned}
& f_{1}\left(x, t_{1}, t_{2}, t_{3}\right) \leq \varepsilon_{0} t_{1}+\lambda t_{2}+\varepsilon_{0} t_{3}^{1 /(p-1)}+c, \\
& f_{2}\left(x, t_{1}, t_{2}, t_{3}\right) \leq \varepsilon_{0} t_{1}^{p-1}+\varepsilon_{0} t_{2}^{p-1}+\mu t_{3}+c, \\
& f_{3}\left(x, t_{1}, t_{2}, t_{3}\right) \leq \gamma \rho(x) t_{1}^{p-1}+\varepsilon_{0} t_{2}^{p-1}+\varepsilon_{0} t_{3}+c,
\end{aligned}
$$

for every $x \in \Omega$ and $t_{1}, t_{2}, t_{3} \geq 0$, where $\varepsilon_{0}>0$ and $c>0$ are constants, $\rho \in L^{\infty}(\Omega)$, $\rho \geq 0, \rho \neq 0, \lambda, \mu, \gamma>0$, and $\lambda^{p-1} \mu \gamma<\Lambda_{1}^{\rho}$.

If we take $\varepsilon_{0}=c=0$ in (6.1), we recover condition (5.29) in the nonradial setting. In this way, (1.1) is included in the following preliminary result if we assume $f(x, t) \leq \gamma \rho(x) t^{p-1}$ for $\gamma<\Lambda_{1}^{\rho}$. Lemma 6.1 can be viewed as a generalization of Theorem 5.3, unfortunately the solution maybe identically zero. Theorem 6.2 is sharper in the sense that it presents a positive solution under suitable additional conditions. 
LEMMA 6.1. There is a constant $\varepsilon_{0}>0$ such that for each $c>0$ and every $f_{i}$ fulfilling the growth conditions (6.1), system (1.11) admits a nonnegative weak solution in $\left(C^{1}(\bar{\Omega})\right)^{3}$.

Proof. Let $X=\left\{u=\left(u_{1}, u_{2}, u_{3}\right) \in(C(\bar{\Omega}))^{3}: u_{1}=u_{2}=u_{3}=0\right.$ on $\left.\partial \Omega\right\}$ be the space endowed with the norm $\|u\|_{X}=\left\|u_{1}\right\|_{C(\bar{\Omega})}+\left\|u_{2}\right\|_{C(\bar{\Omega})}+\left\|u_{3}\right\|_{C(\bar{\Omega})}$. Denote by $C$ the cone of $X$ given by $C=\{u \in X: u \geq 0$ in $\Omega\}$. Consider the mapping $T_{f}=T\left(f_{1}, f_{2}, f_{3}\right):[0,1] \times C \rightarrow C$ defined by $T_{f}(t, u)=v$, where $v=\left(v_{1}, v_{2}, v_{3}\right)$ satisfies

$$
\begin{aligned}
-\Delta v_{1} & =t f_{1}\left(x, u_{1}, u_{2}, u_{3}\right), \\
-\Delta_{p} v_{2} & =t f_{2}\left(x, u_{1}, u_{2}, u_{3}\right) \quad \text { in } \Omega, \\
-\Delta v_{3} & =t f_{3}\left(x, u_{1}, u_{2}, u_{3}\right), \\
v_{1} & =v_{2}=v_{3}=0 \quad \text { on } \partial \Omega .
\end{aligned}
$$

By the maximum principle and $C^{1}$ estimates, $T_{f}$ is a well-defined, continuous, and compact mapping. We claim that there exists a constant $\varepsilon_{0}>0$ such that for each $0<c \leq 1$ and each triplet of nonnegative functions $f=\left(f_{1}, f_{2}, f_{3}\right)$ satisfying (6.1), there is a constant $M_{0}>0$ not depending on $c$ and $f$ such that $\|u\|_{X}<M_{0}$ for every $u \in C$ with $T_{f}(t, u)=u$ for some $t \in[0,1]$. Otherwise, there exist sequences $t_{k} \in[0,1], u_{k} \in C, c_{k} \in(0,1], \varepsilon_{k} \in(0,+\infty)$, and $\left(f_{1 k}, f_{2 k}, f_{3 k}\right)_{k}$ verifying $\varepsilon_{k} \rightarrow 0, T_{k}\left(t_{k}, u_{k}\right)=u_{k},\left\|u_{k}\right\|_{X} \rightarrow \infty$, and (6.1) with $c_{k},\left(f_{1 k}, f_{2 k}, f_{3 k}\right)_{k}$, and $\varepsilon_{k}$ in place of $c,\left(f_{1}, f_{2}, f_{3}\right)$, and $\varepsilon$, respectively, where $T_{k}=T\left(f_{1 k}, f_{2 k}, f_{3 k}\right)$ and $u_{k}=\left(u_{1 k}, u_{2 k}, u_{3 k}\right)$. Define

$$
\begin{aligned}
\tilde{u}_{1 k} & =\frac{u_{1 k}}{\left\|u_{1 k}\right\|_{C(\bar{\Omega})}+\left\|u_{2 k}\right\|_{C(\bar{\Omega})}+\left\|u_{3 k}\right\|_{C(\bar{\Omega})}^{1 /(p-1)}} ; \\
\tilde{u}_{2 k} & =\frac{u_{2 k}}{\left\|u_{1 k}\right\|_{C(\bar{\Omega})}+\left\|u_{2 k}\right\|_{C(\bar{\Omega})}+\left\|u_{3 k}\right\|_{C(\bar{\Omega})}^{1 /(p-1)}} ; \\
\tilde{u}_{3 k} & =\frac{u_{3 k}}{\left(\left\|u_{1 k}\right\|_{C(\bar{\Omega})}+\left\|u_{2 k}\right\|_{C(\bar{\Omega})}+\left\|u_{3 k}\right\|_{C(\bar{\Omega})}^{1 /(p-1)}\right)^{p-1}} .
\end{aligned}
$$

Then $\left\|\tilde{u}_{1 k}\right\|_{C(\bar{\Omega})}+\left\|\tilde{u}_{2 k}\right\|_{C(\bar{\Omega})}+\left\|\tilde{u}_{3 k}\right\|_{C(\bar{\Omega})}^{1 /(p-1)}=1$. Using (6.1) and applying $C^{1}$ estimates in (6.2), up to a subsequence, we conclude that $\tilde{u}_{k}$ converges to a function $\tilde{u}$ in $\left(C^{1}(\bar{\Omega})\right)^{3}$. Furthermore, it follows that $\tilde{u} \geq 0$ in $\Omega,\left\|\tilde{u}_{1}\right\|_{C(\bar{\Omega})}+\left\|\tilde{u}_{2}\right\|_{C(\bar{\Omega})}+$ $\left\|\tilde{u}_{3}\right\|_{C(\bar{\Omega})}^{1 /(p-1)}=1$, and

$$
\begin{aligned}
-\Delta \tilde{u}_{1} & =\tilde{f}_{1}(x) \leq \lambda \tilde{u}_{2}, \\
-\Delta_{p} \tilde{u}_{2} & =\tilde{f}_{2}(x) \leq \mu \tilde{u}_{3} \quad \text { in } \Omega, \\
-\Delta \tilde{u}_{3} & =\tilde{f}_{3}(x) \leq \gamma \rho(x) \tilde{u}_{1}^{p-1}, \\
\tilde{u}_{1} & =\tilde{u}_{2}=\tilde{u}_{3}=0 \quad \text { on } \partial \Omega,
\end{aligned}
$$


where each $\tilde{f}_{i}$ is a nonnegative function belonging to $L^{\beta}(\Omega), \beta>1$. Since $\tilde{u}_{i}>0$ in $\Omega$ for some $i=1,2,3$, by Proposition 3.2(ii), we get a contradiction. Thus, the claimed constants $\varepsilon_{0}>0$ and $M_{0}>0$ do exist. Choose an arbitrary number $c>0$ and a triplet of nonnegative functions $f=\left(f_{1}, f_{2}, f_{3}\right)$ satisfying (6.1) with $\varepsilon_{0}$ provided above. We affirm that there is a constant $M=M(c)>0$ not depending on $f$ such that $\|u\|_{X}<M$ for every $u \in C$ with $T_{f}(t, u)=u$ for some $t \in[0,1]$. In fact, for each $\varepsilon>0$ define the functions $f_{1 \varepsilon}\left(x, t_{1}, t_{2}, t_{3}\right)=\varepsilon f_{1}\left(x, t_{1} / \varepsilon, t_{2} / \varepsilon, t_{3} / \varepsilon^{p-1}\right)$, $f_{2 \varepsilon}\left(x, t_{1}, t_{2}, t_{3}\right)=\varepsilon^{p-1} f_{2}\left(x, t_{1} / \varepsilon, t_{2} / \varepsilon, t_{3} / \varepsilon^{p-1}\right), f_{3 \varepsilon}\left(x, t_{1}, t_{2}, t_{3}\right)=\varepsilon^{p-1} f_{3}\left(x, t_{1} / \varepsilon, t_{2} / \varepsilon\right.$, $\left.t_{3} / \varepsilon^{p-1}\right)$ and put $f_{\varepsilon}=\left(f_{1 \varepsilon}, f_{2 \varepsilon}, f_{3 \varepsilon}\right)$. Clearly, $f_{\varepsilon}$ fulfills (6.1) with $c_{\varepsilon}=\max \left\{\varepsilon^{p-1}, \varepsilon\right\} c$ instead of $c$. Since the functions $u_{1 \varepsilon}=\varepsilon u_{1}, u_{2 \varepsilon}=\varepsilon u_{2}$, and $u_{3 \varepsilon}=\varepsilon^{p-1} u_{3}$ verify $T_{f_{\varepsilon}}\left(t, u_{\varepsilon}\right)=u_{\varepsilon}$, taking $\varepsilon$ small enough such that $0<c_{\varepsilon} \leq 1$, from the first part, it follows that $\left\|u_{\varepsilon}\right\|_{X}<M_{0}$. Therefore, we conclude that $\|u\|_{X}<M$. Hence, by the homotopic invariance property of the degree in cones, we obtain $\operatorname{deg}(I-$ $\left.T_{f}(1, \cdot), B_{M} \cap C, 0\right)=\operatorname{deg}\left(I-T_{f}(0, \cdot), B_{M} \cap C, 0\right) \neq 0$, implying that $T_{f}(1, u)=u$ for some $u \in\left(C^{1}(\bar{\Omega})\right)^{3} \cap C$.

The above lemma is not useful to seek nontrivial weak solutions when $\left(u_{1}, u_{2}, u_{3}\right) \equiv 0$ in $\Omega$ solves problem (1.11). Further assumptions will lead us to find a positive solution of (1.11). We assume that

$$
\begin{aligned}
& f_{1}\left(x, t_{1}, t_{2}, t_{3}\right) \geq \lambda_{0} t_{2}, \\
& f_{2}\left(x, t_{1}, t_{2}, t_{3}\right) \geq \mu_{0} t_{3}, \\
& f_{3}\left(x, t_{1}, t_{2}, t_{3}\right) \geq \gamma_{0} \rho_{0}(x) t_{1}^{p-1},
\end{aligned}
$$

for every $x \in \Omega$ and $0<t_{1}, t_{2}, t_{3} \leq \delta_{0}$, where $\delta_{0}>0$ is a constant, $\rho_{0} \in L^{\infty}(\Omega)$, $\rho_{0} \geq 0, \rho_{0} \neq 0, \lambda_{0}, \mu_{0}, \gamma_{0}>0$, and $\lambda_{0}^{p-1} \mu_{0} \gamma_{0}=\Lambda_{1}^{\rho_{0}}$.

Notice that $\gamma_{0}$ can be equal to $\Lambda_{1}^{\rho}$. Thus in the framework of (1.1), condition (6.5) reduces to $f(x, t) \geq \Lambda_{1}^{\rho} \rho(x) t^{p-1}$ for small $t$.

We also suppose some monotonicity on the functions $f_{i}$, namely

$$
f_{1}\left(x, t_{1}, t_{2}, t_{3}\right) \leq f_{1}\left(x, s_{1}, s_{2}, s_{3}\right)
$$

for $x \in \Omega, 0 \leq t_{1}=s_{1} \leq \delta, 0 \leq t_{2} \leq s_{2}$, and $0 \leq t_{3} \leq s_{3}$,

$$
f_{2}\left(x, t_{1}, t_{2}, t_{3}\right) \leq f_{2}\left(x, s_{1}, s_{2}, s_{3}\right)
$$

for $x \in \Omega, 0 \leq t_{1} \leq s_{1}, 0 \leq t_{2}=s_{2} \leq \delta$, and $0 \leq t_{3} \leq s_{3}$,

$$
f_{3}\left(x, t_{1}, t_{2}, t_{3}\right) \leq f_{3}\left(x, s_{1}, s_{2}, s_{3}\right)
$$

for $x \in \Omega, 0 \leq t_{1} \leq s_{1}, 0 \leq t_{2} \leq s_{2}$, and $0 \leq t_{3}=s_{3} \leq \delta$, where $\delta>0$.

We establish the following result by performing a truncation between a subsolution and supersolution. 
THEOREM 6.2. System (1.11) admits a positive weak solution in $\left(C^{1}(\bar{\Omega})\right)^{3}$ if we assume (6.1) with $\varepsilon_{0}>0$ given in Lemma 6.1 and conditions (6.5), (6.6), (6.7), and (6.8).

Proof. We first show that problem (1.11) possesses a positive subsolution. In fact, denote by $\left(v_{1}, v_{2}, v_{3}\right)$ a positive eigenfunction corresponding to $\left(\lambda_{0}, \mu_{0}, \gamma_{0}\right)$. Since $\left(t v_{1}, t v_{2}, t^{p-1} v_{3}\right)$ is also an eigenfunction, we can assume $v_{i} \leq \min \left\{\delta, \delta_{0}\right\}$ in $\Omega$ for $i=1,2,3$. From (6.5), we conclude that $\left(v_{1}, v_{2}, v_{3}\right)$ is a positive subsolution of system (1.11). We prove now that the problem has a positive solution. Define for $i=1,2,3$ the Carathéodory functions

$$
F_{i}\left(x, t_{1}, t_{2}, t_{3}\right)= \begin{cases}f_{i}\left(x, t_{1}, t_{2}, t_{3}\right) & \text { if } t_{1} \geq v_{1}(x), t_{2} \geq v_{2}(x), t_{3} \geq v_{3}(x), \\ f_{i}\left(x, t_{1}, t_{2}, v_{3}(x)\right) & \text { if } t_{1} \geq v_{1}(x), t_{2} \geq v_{2}(x), t_{3}<v_{3}(x), \\ f_{i}\left(x, t_{1}, v_{2}(x), t_{3}\right) & \text { if } t_{1} \geq v_{1}(x), t_{2}<v_{2}(x), t_{3} \geq v_{3}(x), \\ f_{i}\left(x, v_{1}(x), t_{2}, t_{3}\right) & \text { if } t_{1}<v_{1}(x), t_{2} \geq v_{2}(x), t_{3} \geq v_{3}(x), \\ f_{i}\left(x, t_{1}, v_{2}(x), v_{3}(x)\right) & \text { if } t_{1} \geq v_{1}(x), t_{2}<v_{2}(x), t_{3}<v_{3}(x), \\ f_{i}\left(x, v_{1}(x), t_{2}, v_{3}(x)\right) & \text { if } t_{1}<v_{1}(x), t_{2} \geq v_{2}(x), t_{3}<v_{3}(x), \\ f_{i}\left(x, v_{1}(x), v_{2}(x), t_{3}\right), & \text { if } t_{1}<v_{1}(x), t_{2}<v_{2}(x), t_{3} \geq v_{3}(x), \\ f_{i}\left(x, v_{1}(x), v_{2}(x), v_{3}(x)\right) & \text { if } t_{1}<v_{1}(x), t_{2}<v_{2}(x), t_{3}<v_{3}(x),\end{cases}
$$

Clearly, each $F_{i}$ verifies condition (6.1) for some sufficiently large $c>0$. Lemma 6.1 implies that the system

$$
\begin{aligned}
-\Delta u_{1} & =F_{1}\left(x, u_{1}, u_{2}, u_{3}\right), \\
-\Delta_{p} u_{2} & =F_{2}\left(x, u_{1}, u_{2}, u_{3}\right) \quad \text { in } \Omega, \\
-\Delta u_{3} & =F_{3}\left(x, u_{1}, u_{2}, u_{3}\right), \\
u_{1} & =u_{2}=u_{3}=0 \quad \text { on } \partial \Omega
\end{aligned}
$$

admits a nonnegative solution $\left(u_{1}, u_{2}, u_{3}\right) \in\left(C^{1}(\bar{\Omega})\right)^{3}$. We claim that $u_{1} \geq v_{1}$ in $\Omega$. Otherwise, $\Omega^{-}=\left\{x \in \Omega: u_{1}(x)<v_{1}(x)\right\}$ is a nonempty open subset of $\Omega$. Given $x \in \Omega^{-}$, consider the difference $d=f_{1}\left(x, v_{1}(x), v_{2}(x), v_{3}(x)\right)-F_{1}\left(x, u_{1}(x)\right.$, $\left.u_{2}(x), u_{3}(x)\right)$. There are four cases to be analyzed:

(i) if $u_{2}(x) \geq v_{2}(x)$ and $u_{3}(x) \geq v_{3}(x)$. Since $F_{1}\left(x, u_{1}, u_{2}, u_{3}\right)=f_{1}\left(x, v_{1}, u_{2}\right.$, $\left.u_{3}\right)$, from (6.6), we get $d \leq 0$;

(ii) if $u_{2}(x)<v_{2}(x)$ and $u_{3}(x) \geq v_{3}(x)$, again since $F_{1}\left(x, u_{1}, u_{2}, u_{3}\right)=f_{1}\left(x, v_{1}\right.$, $\left.v_{2}, u_{3}\right)$, from $(6.6)$, we obtain $d \leq 0$;

(iii) if $u_{2}(x) \geq v_{2}(x)$ and $u_{3}(x)<v_{3}(x)$, a similar reasoning to the second case furnishes the conclusion;

(iv) if $u_{2}(x)<v_{2}(x)$ and $u_{3}(x)<v_{3}(x)$, by definition of $d$, it follows that $d=0$.

Therefore, $f_{1}\left(x, v_{1}(x), v_{2}(x), v_{3}(x)\right)-F_{1}\left(x, u_{1}(x), u_{2}(x), u_{3}(x)\right) \leq 0$ for every $x$ $\in \Omega^{-}$, implying $\Delta\left(u_{1}-v_{1}\right) \leq 0$ in $\Omega^{-}$. Since $u_{1}=v_{1}$ on $\partial \Omega^{-}$, using the maximum principle, we conclude that $u_{1} \geq v_{1}$ in $\Omega^{-}$, a contradiction. So, $u_{1} \geq v_{1}$ 
in $\Omega$. By similar ideas and Lemma 3.1, we show that $u_{2} \geq v_{2}$ and $u_{3} \geq v_{3}$ in $\Omega$. Consequently, by definition of $F_{i}, i=1,2,3$, the triplet $\left(u_{1}, u_{2}, u_{3}\right)$ is a positive solution of system (1.11).

Under somewhat different conditions, it is possible to obtain a positive solution again. The following two requirements are modifications of (6.1). We rewrite assumption (6.1) below

$$
\begin{aligned}
& f_{1}\left(x, t_{1}, t_{2}, t_{3}\right) \leq a\left(t_{1}+t_{2}+t_{3}^{1 /(p-1)}+1\right), \\
& f_{2}\left(x, t_{1}, t_{2}, t_{3}\right) \leq a\left(t_{1}^{p-1}+t_{2}^{p-1}+t_{3}+1\right), \\
& f_{3}\left(x, t_{1}, t_{2}, t_{3}\right) \leq a\left(t_{1}^{p-1}+t_{2}^{p-1}+t_{3}+1\right)
\end{aligned}
$$

for some constant $a>0$ and every $x \in \Omega$ and $t_{1}, t_{2}, t_{3} \geq 0$.

Taking $\varepsilon_{0}=c=0$ in (6.1), we obtain

$$
\begin{aligned}
& f_{1}\left(x, t_{1}, t_{2}, t_{3}\right) \leq \lambda_{0} t_{2}, \\
& f_{2}\left(x, t_{1}, t_{2}, t_{3}\right) \leq \mu_{0} t_{3}, \\
& f_{3}\left(x, t_{1}, t_{2}, t_{3}\right) \leq \gamma_{0} \rho_{0}(x) t_{1}^{p-1},
\end{aligned}
$$

for every $x \in \Omega$ and $0<t_{1}, t_{2}, t_{3} \leq \delta_{0}$, where $\delta_{0}>0$ is a constant, $\rho_{0} \in L^{\infty}(\Omega)$, $\rho_{0} \neq \equiv, \rho_{0} \geq 0, \lambda_{0}, \mu_{0}, \gamma_{0}>0, \lambda_{0}^{p-1} \mu_{0} \gamma_{0}<\Lambda_{1}^{\rho_{0}}$.

The following condition is a kind of nonresonance:

$$
\begin{aligned}
& f_{1}\left(x, t_{1}, t_{2}, t_{3}\right) \geq \lambda t_{2}-c, \\
& f_{2}\left(x, t_{1}, t_{2}, t_{3}\right) \geq \mu t_{3}-c, \\
& f_{3}\left(x, t_{1}, t_{2}, t_{3}\right) \geq \gamma \rho(x) t_{1}^{p-1}-c,
\end{aligned}
$$

for every $x \in \Omega$ and $t_{1}, t_{2}, t_{3} \geq 0$, where $c>0$ is a constant, $\rho \in L^{\infty}(\Omega), \rho \geq 0$, $\rho \neq 0, \lambda, \mu, \gamma>0$, and $\lambda^{p-1} \mu \gamma>\Lambda_{1}^{\rho}$.

It is easy to see that the following result applies to (1.1); observe the difference between (6.5) and conditions (6.12) and (6.13).

THEOREM 6.3. System (1.11) admits a nontrivial nonnegative weak solution in $\left(C^{1}(\bar{\Omega})\right)^{3}$, provided that (6.11), (6.12), and (6.13) are verified.

Proof. Let $X$ and $C$ be as in the proof of Lemma 6.1. Define the mapping $H$ : $[0,+\infty) \times C \rightarrow C$ by $H(t, u)=v$, where $v=\left(v_{1}, v_{2}, v_{3}\right)$ verifies

$$
\begin{aligned}
-\Delta v_{1} & =f_{1}\left(x, u_{1}, u_{2}, u_{3}\right)+t, \\
-\Delta_{p} v_{2} & =f_{2}\left(x, u_{1}, u_{2}, u_{3}\right)+t \quad \text { in } \Omega, \\
-\Delta v_{3} & =f_{3}\left(x, u_{1}, u_{2}, u_{3}\right)+t, \\
v_{1} & =v_{2}=v_{3}=0 \quad \text { on } \partial \Omega .
\end{aligned}
$$


By the maximum principle and $C^{1}$ estimates, it follows that $H$ is well defined, continuous, compact, and $H(0,0)=0$. Let $T: C \rightarrow C$ be given by $T(u)=H(0, u)$. At first, we get $r>0$ such that $u \neq t T(u)$, for every $t \in[0,1]$ and $u \in C$ with $\|u\|_{X}=r$. In fact, take $0<r \leq \delta_{0}$ and suppose $u=t T(u)$ for some $t \in[0,1]$ and $u \in C$ with $\|u\|_{X}=r$. Then, from (6.12), we get

$$
\begin{aligned}
-\Delta u_{1} & =t f_{1}\left(x, u_{1}, u_{2}, u_{3}\right) \leq \lambda_{0} u_{2}, \\
-\Delta_{p} u_{2} & =t^{p-1} f_{2}\left(x, u_{1}, u_{2}, u_{3}\right) \leq \mu_{0} u_{3} \quad \text { in } \Omega, \\
-\Delta u_{3} & =t f_{3}\left(x, u_{1}, u_{2}, u_{3}\right) \leq \gamma_{0} \rho_{0}(x) u_{1}^{p-1} .
\end{aligned}
$$

In particular, one of the components of $u$ is positive in $\Omega$. By virtue of Proposition 3.2(ii), we get a contradiction. We prove that there exist $R>r$ and $t_{0}>0$ such that $H(t, u) \neq u$ for every $t \geq t_{0}$ and $u \in C$ with $\|u\|_{X} \leq R$ and also for every $t \geq 0$ when $\|u\|_{X}=R$. Indeed, let $t \geq 0$ and $u \in C$ with $H(t, u)=u$. From (6.13), we have

$$
\begin{aligned}
-\Delta u_{1} & \geq \lambda u_{2}+t-c, \\
-\Delta_{p} u_{2} & \geq \mu u_{3}+t-c \quad \text { in } \Omega, \\
-\Delta u_{3} & \geq \gamma \rho(x) u_{1}^{p-1}+t-c .
\end{aligned}
$$

By Proposition 3.2(iii), we see that $t \leq c$. Hence, we can take $R>0$ such that $\|u\|_{X} \leq R$ for every $u \in C$ verifying $H(t, u)=u$ for some $t \in[0, c]$. Otherwise, there are sequences $t_{k} \in[0, c]$ and $u_{k} \in C$ satisfying $H\left(t_{k}, u_{k}\right)=u_{k}$ and $\left\|u_{k}\right\|_{X} \rightarrow$ $\infty$. Denoting $\tilde{u}_{i k}$ the normalized functions as in the proof of Lemma 6.1, we have $\left\|\tilde{u}_{1 k}\right\|_{C(\bar{\Omega})}+\left\|\tilde{u}_{2 k}\right\|_{C(\bar{\Omega})}+\left\|\tilde{u}_{3 k}\right\|_{C(\bar{\Omega})}^{1 /(p-1)}=1$. Using (6.11), (6.13), and $C^{1}$ estimates, up to a subsequence, we conclude that $\tilde{u}_{k}$ converges to a function $\tilde{u}$ in $\left(C^{1}(\bar{\Omega})\right)^{3}$. In particular, $\tilde{u} \geq 0$ in $\Omega,\left\|\tilde{u}_{1}\right\|_{C(\bar{\Omega})}+\left\|\tilde{u}_{2}\right\|_{C(\bar{\Omega})}+\left\|\tilde{u}_{3}\right\|_{C(\bar{\Omega})}^{1 /(p-1)}=1$, and

$$
\begin{aligned}
-\Delta \tilde{u}_{1} & \geq \lambda \tilde{u}_{2}, \\
-\Delta_{p} \tilde{u}_{2} & \geq \mu \tilde{u}_{3} \quad \text { in } \Omega, \\
-\Delta \tilde{u}_{3} & \geq \gamma \rho(x) \tilde{u}_{1}^{p-1}, \\
\tilde{u}_{1}=\tilde{u}_{2} & =\tilde{u}_{3}=0 \quad \text { on } \partial \Omega .
\end{aligned}
$$

Thus, $\tilde{u}>0$ in $\Omega$, contradicting (iii) of Proposition 3.2. Hence, the conclusion follows from Krasnosel'skii theorem (Theorem 5.2).

\section{Uniqueness of solutions}

In this section, we give conditions under which problem (1.11) admits a unique positive weak solution. Essentially, we assume a certain homogeneity and 
monotonicity on functions $f_{i}$

$$
\begin{aligned}
& f_{1}\left(x, s t_{1}, s t_{2}, s^{p-1} t_{3}\right) \geq s f_{1}\left(x, t_{1}, t_{2}, t_{3}\right), \\
& f_{2}\left(x, s t_{1}, s t_{2}, s^{p-1} t_{3}\right) \geq s^{p-1} f_{2}\left(x, t_{1}, t_{2}, t_{3}\right), \\
& f_{3}\left(x, s t_{1}, s t_{2}, s^{p-1} t_{3}\right) \geq s^{p-1} f_{3}\left(x, t_{1}, t_{2}, t_{3}\right),
\end{aligned}
$$

for every $x \in \Omega, s \in[0,1]$, and $t_{1}, t_{2}, t_{3}>0$,

$$
f_{i}\left(x, t_{1}, t_{2}, t_{3}\right) \leq f_{i}\left(x, s_{1}, s_{2}, s_{3}\right)
$$

for every $x \in \Omega, 0<t_{1} \leq s_{1}, 0<t_{2} \leq s_{2}, 0<t_{3} \leq s_{3}$, and $i=1,2,3$,

$$
f_{1}\left(x_{1}, t_{1}, t_{2}, t_{3}\right)<f_{1}\left(x_{1}, s_{1}, s_{2}, s_{3}\right)
$$

for some $x_{1} \in \Omega$ and every $0<t_{1} \leq s_{1}, 0<t_{2}<s_{2}, 0<t_{3} \leq s_{3}$,

$$
f_{2}\left(x, t_{1}, t_{2}, t_{3}\right)<f_{2}\left(x, s_{1}, s_{2}, s_{3}\right),
$$

for every $x$ in a neighborhood of $\partial \Omega, 0<t_{1} \leq s_{1}, 0<t_{2} \leq s_{2}, 0<t_{3}<s_{3}$, and

$$
f_{3}\left(x_{3}, t_{1}, t_{2}, t_{3}\right)<f_{3}\left(x_{3}, s_{1}, s_{2}, s_{3}\right)
$$

for some $x_{3} \in \Omega$ and every $0<t_{1}<s_{1}, 0<t_{2} \leq s_{2}, 0<t_{3} \leq s_{3}$.

We adopt a variant of a comparison strategy due to Krasnosel'skiü [5], see also [12].

THEOREM 7.1. System (1.11) admits, at most, one positive weak solution in $\left(C^{1}(\bar{\Omega})\right)^{3}$, provided (7.1), (7.2), (7.3), (7.4), (7.5), (7.6), and (7.7) are true and if just only one of inequalities (7.1) or (7.3) holds in the strict sense for some $x_{0} \in \Omega$ and every $s \in(0,1)$.

Proof. Let $\left(u_{1}, u_{2}, u_{3}\right)$ and $\left(v_{1}, v_{2}, v_{3}\right)$ be two positive weak solutions of (1.11) belonging to $\left(C^{1}(\bar{\Omega})\right)^{3}$. Define the set $S=\left\{s>0: v_{1}>s u_{1}, v_{2}>s u_{2}\right.$ and $v_{3}>$ $s^{p-1} u_{3}$ in $\left.\Omega\right\}$. Take $s^{*}=\sup S$. Changing $\left(u_{1}, u_{2}, u_{3}\right)$ and $\left(v_{1}, v_{2}, v_{3}\right)$, if necessary, we may assume that $s^{*} \in(0,1]$. We show first that $v_{2}(y)=s^{*} u_{2}(y)$ for some $y \in \Omega$. Indeed, suppose on the contrary that $v_{2}>s^{*} u_{2}$ in $\Omega$. Since

$$
\begin{aligned}
-\Delta_{p} v_{2}+\Delta_{p} s^{*} u_{2} & =f_{2}\left(x, v_{1}, v_{2}, v_{3}\right)-s^{* p-1} f_{2}\left(x, u_{1}, u_{2}, u_{3}\right) \\
& \geq f_{2}\left(x, s^{*} u_{1}, s^{*} u_{2}, s^{*-1} u_{3}\right)-s^{* p^{-1}} f_{2}\left(x, u_{1}, u_{2}, u_{3}\right) \\
& \geq 0
\end{aligned}
$$


then, by Lemma 3.1, $v_{2}>\left(s^{*}+\varepsilon\right) u_{2}$ in $\Omega$ for $\varepsilon>0$ small enough. Thus,

$$
\begin{aligned}
-\Delta\left(v_{1}-s^{*} u_{1}\right) & =f_{1}\left(x, v_{1}, v_{2}, v_{3}\right)-s^{*} f_{1}\left(x, u_{1}, u_{2}, u_{3}\right) \\
& \geq f_{1}\left(x, s^{*} u_{1}, s^{*} u_{2}, s^{*-1} u_{3}\right)-s^{*} f_{1}\left(x, u_{1}, u_{2}, u_{3}\right) \\
& \geq 0 \\
-\Delta\left(v_{1}-s^{*} u_{1}\right)\left(x_{1}\right) & =f_{1}\left(x_{1}, v_{1}, v_{2}, v_{3}\right)-s^{*} f_{1}\left(x_{1}, u_{1}, u_{2}, u_{3}\right) \\
& >f_{1}\left(x_{1}, s^{*} u_{1}, s^{*} u_{2}, s^{*-1} u_{3}\right)-s^{*} f_{1}\left(x_{1}, u_{1}, u_{2}, u_{3}\right) \\
& \geq 0
\end{aligned}
$$

imply that $v_{1}>\left(s^{*}+\varepsilon\right) u_{1}$ in $\Omega$ for $\varepsilon>0$ small enough. The inequalities

$$
\begin{aligned}
-\Delta\left(v_{3}-s^{* p-1} u_{3}\right) & =f_{3}\left(x, v_{1}, v_{2}, v_{3}\right)-s^{*-1} f_{3}\left(x, u_{1}, u_{2}, u_{3}\right) \\
& \geq f_{3}\left(x, s^{*} u_{1}, s^{*} u_{2}, s^{*-1} u_{3}\right)-s^{*-1} f_{3}\left(x, u_{1}, u_{2}, u_{3}\right) \\
& \geq 0, \\
-\Delta\left(v_{3}-s^{* p-1} u_{3}\right)\left(x_{3}\right) & =f_{3}\left(x_{3}, v_{1}, v_{2}, v_{3}\right)-s^{*-1} f_{3}\left(x_{3}, u_{1}, u_{2}, u_{3}\right) \\
& >f_{3}\left(x_{3}, s^{*} u_{1}, s^{*} u_{2}, s^{*-1} u_{3}\right)-s^{*-1} f_{3}\left(x_{3}, u_{1}, u_{2}, u_{3}\right) \\
& \geq 0
\end{aligned}
$$

furnish $v_{3}>\left(s^{*}+\varepsilon\right)^{p-1} u_{3}$ in $\Omega$ for $\varepsilon>0$ small enough, contradicting the definition of $s^{*}$. Hence, there is $y \in \Omega$ satisfying $v_{2}(y)=s^{*} u_{2}(y)$. Choose the set $\Gamma_{2}$ associated to the function $v_{2}$ as in Lemma 3.1. We show next that there exists $z \in \Gamma_{2}$ such that $v_{2}(z)=s^{*} u_{2}(z)$. Take a subdomain $\Omega_{0}$ of $\Omega$ with smooth boundary $\partial \Omega_{0}$ which verifies $\bar{\Omega}_{0} \subset \Omega, \partial \Omega_{0} \subset \Gamma_{2}$, and $y \in \Omega_{0}$. We claim that there is $z \in \partial \Omega_{0}$ with $v_{2}(z)=s^{*} u_{2}(z)$. Indeed, if $v_{2}>s^{*} u_{2}$ on $\partial \Omega_{0}$, by continuity, we get $v_{2} \geq s^{*} u_{2}+\eta$ on $\partial \Omega_{0}$ for some $\eta>0$. Since $-\Delta_{p} v_{2} \geq-\Delta_{p} s^{*} u_{2}=-\Delta_{p}\left(s^{*} u_{2}+\eta\right)$ in $\Omega_{0}$, then by Lemma 3.1, $v_{2} \geq s^{*} u_{2}+\eta$ in $\Omega_{0}$. But $y \in \Omega_{0}$, so we arrive at a contradiction. Therefore, the claimed point $z \in \Gamma_{2}$ exists. Noting that $-\Delta_{p} v_{2} \geq$ $-\Delta_{p} s^{*} u_{2}$ in $\Gamma_{2}, v_{2} \geq s^{*} u_{2}$ in $\Gamma_{2}$ and $v_{2}(z)=s^{*} u_{2}(z)$. It follows that $v_{2} \equiv s^{*} u_{2}$ in $\Gamma_{2}$, again by Lemma 3.1. We affirm that $v_{1} \equiv s^{*} u_{1}$ and $v_{3} \equiv s^{* p^{-1}} u_{3}$ in $\Omega$. In fact, suppose $v_{1} \not \equiv s^{*} u_{1}$ in $\Omega$. Using (7.1), (7.4), and (7.5) and the strong maximum principle as above, we conclude that $v_{1}>s^{*} u_{1}$ in $\Omega$. Applying (7.1), (7.4), (7.7), and the strong maximum principle once more, it follows easily that $v_{3}>s^{* p^{-1}} u_{3}$ in $\Omega$. Finally, from condition (7.6), there is a point $x_{2} \in \Gamma_{2}$ such that

$$
\begin{aligned}
-\Delta_{p} v_{2}\left(x_{2}\right)+\Delta_{p}\left(s^{*} u_{2}\right)\left(x_{2}\right) & =f_{2}\left(x_{2}, v_{1}, v_{2}, v_{3}\right)-s^{* p^{-1}} f_{2}\left(x_{2}, u_{1}, u_{2}, u_{3}\right) \\
& >f_{2}\left(x_{2}, s^{*} u_{1}, s^{*} u_{2}, s^{*-1} u_{3}\right)-s^{*-1} f_{2}\left(x_{2}, u_{1}, u_{2}, u_{3}\right) \\
& \geq 0
\end{aligned}
$$


contradicting $v_{2} \equiv s^{*} u_{2}$ in $\Gamma$. Similarly, we see that $v_{3} \equiv s^{*{ }^{p-1}} u_{3}$ in $\Omega$. We prove that $s^{*}=1$. Indeed, assume that $s^{*} \in(0,1)$. If (7.3) holds strictly for some $x_{0} \in$ $\Omega$, that is,

$$
f_{3}\left(x_{0}, s t_{1}, s t_{2}, s^{p-1} t_{3}\right)>s^{p-1} f_{3}\left(x_{0}, t_{1}, t_{2}, t_{3}\right)
$$

for every $s \in(0,1)$ and $t_{1}, t_{2}, t_{3}>0$, then

$$
\begin{aligned}
-\Delta\left(v_{3}-s^{*-1} u_{3}\right)\left(x_{0}\right) & =f_{3}\left(x_{0}, v_{1}, v_{2}, v_{3}\right)-s^{* p-1} f_{3}\left(x_{0}, u_{1}, u_{2}, u_{3}\right) \\
& =f_{3}\left(x_{0}, s^{*} u_{1}, s^{*} u_{2}, s^{*-1} u_{3}\right)-s^{*-1} f_{3}\left(x_{0}, u_{1}, u_{2}, u_{3}\right) \\
& >0,
\end{aligned}
$$

a contradiction. If (7.1) holds strictly in some point of $\Omega$, we proceed analogously. Therefore, we have $s^{*}=1$. Define the set $\tilde{S}=\left\{s>0: u_{1}>s v_{1}, u_{2}>\right.$ $s v_{2}$ and $u_{3}>s^{p-1} v_{3}$ in $\left.\Omega\right\}$ and let $\tilde{s}=\sup \tilde{S}$. Since $s^{*} \tilde{s} \leq 1$, then $\tilde{s} \leq 1$. Taking the set $\Gamma_{2}$ smaller if necessary and arguing in a similar manner, we conclude that $u_{2} \equiv \tilde{s} v_{2}$ in $\Gamma_{2}$. Hence $s^{*}=\tilde{s}=1$ and as a consequence, we have $u_{1} \equiv v_{1}, u_{2} \equiv v_{2}$, and $u_{3} \equiv v_{3}$ in $\Omega$.

\section{Acknowledgment}

The author was partially supported by FAPESP.

\section{References}

[1] A. Anane, Simplicité et isolation de la première valeur propre du p-Laplacien avec poids [Simplicity and isolation of the first eigenvalue of the p-Laplacian with weight], C. R. Acad. Sci. Paris Sér. I Math. 305 (1987), no. 16, 725-728 (French).

[2] P. Clément, J. Fleckinger, E. Mitidieri, and F. de Thélin, Existence of positive solutions for a nonvariational quasilinear elliptic system, J. Differential Equations 166 (2000), no. 2, 455-477.

[3] P. Clément, R. Manásevich, and E. Mitidieri, Positive solutions for a quasilinear system via blow up, Comm. Partial Differential Equations 18 (1993), no. 12, 2071-2106.

[4] H.-C. Grunau and G. Sweers, Positivity properties of elliptic boundary value problems of higher order, Nonlinear Anal. 30 (1997), no. 8, 5251-5258.

[5] M. A. Krasnosel'skiú, Positive Solutions of Operator Equations, P. Noordhoff Ltd., Groningen, 1964, translated from the Russian by Flaherty, R. E.

[6] G. Lu, J. Wei, and $\mathrm{X}$. Xu, On conformally invariant equation $(-\Delta)^{p} u-$ $K(x) u^{N+2 p / N-2 p}=0$ and its generalizations, Ann. Mat. Pura Appl. (4) 179 (2001), 309-329.

[7] S. Luckhaus, Existence and regularity of weak solutions to the Dirichlet problem for semilinear elliptic systems of higher order, J. reine angew. Math. 306 (1979), 192207.

[8] M. Ôtani, Existence and nonexistence of nontrivial solutions of some nonlinear degenerate elliptic equations, J. Funct. Anal. 76 (1988), no. 1, 140-159. 
[9] L. A. Peletier and R. C. A. M. van der Vorst, Existence and nonexistence of positive solutions of nonlinear elliptic systems and the biharmonic equation, Differential Integral Equations 5 (1992), no. 4, 747-767.

[10] P. Pucci and J. Serrin, A general variational identity, Indiana Univ. Math. J. 35 (1986), no. 3, 681-703.

[11] Critical exponents and critical dimensions for polyharmonic operators, J. Math. Pures Appl. (9) 69 (1990), no. 1, 55-83.

[12] S. Sakaguchi, Concavity properties of solutions to some degenerate quasilinear elliptic Dirichlet problems, Ann. Scuola Norm. Sup. Pisa Cl. Sci. (4) 14 (1987), no. 3, $403-$ 421.

[13] J. L. Vázquez, A strong maximum principle for some quasilinear elliptic equations, Appl. Math. Optim. 12 (1984), no. 3, 191-202.

[14] X. J. Wang, Sharp constant in a Sobolev inequality, Nonlinear Anal. 20 (1993), no. 3, 261-268.

[15] J. Wei and X. Xu, Classification of solutions of higher order conformally invariant equations, Math. Ann. 313 (1999), no. 2, 207-228.

Marcelo Montenegro: Universidade Estadual de Campinas, imeCC, Departamento de Matemática, Caixa Postal 6065, CEP 130813-970, Campinas, SP, Brazil

E-mail address: msm@ime.unicamp.br 


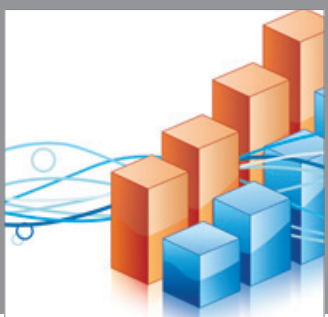

Advances in

Operations Research

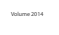

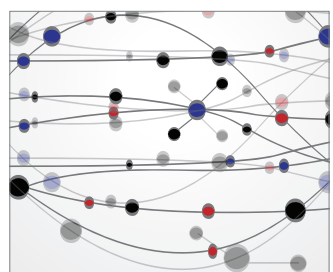

\section{The Scientific} World Journal
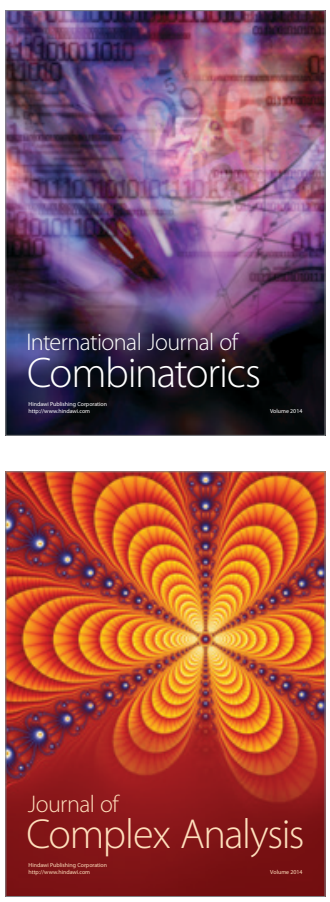

International Journal of

Mathematics and

Mathematical

Sciences
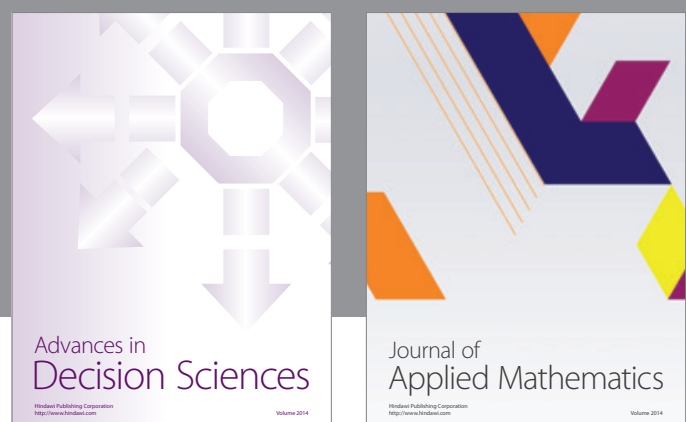

Journal of

Applied Mathematics
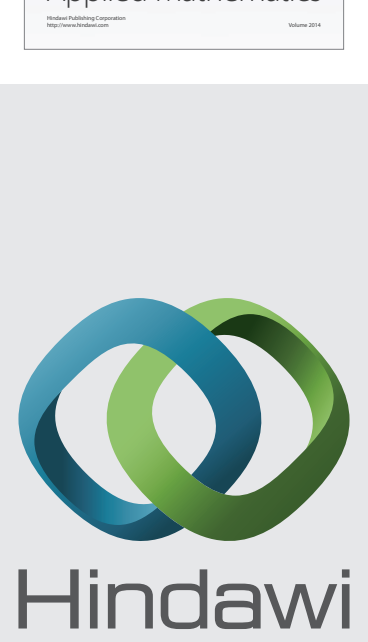

Submit your manuscripts at http://www.hindawi.com
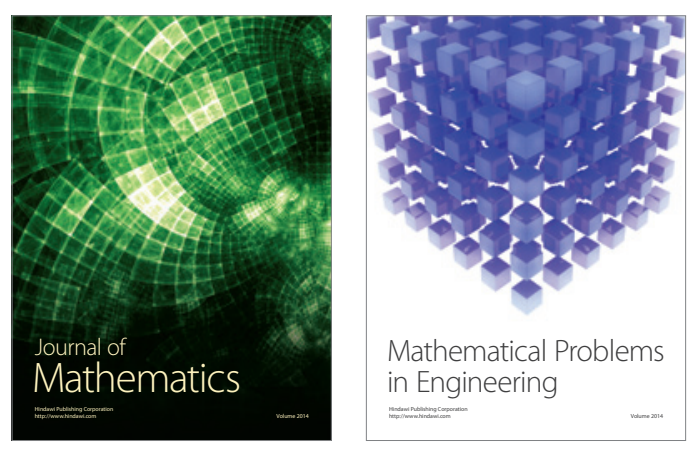

Mathematical Problems in Engineering
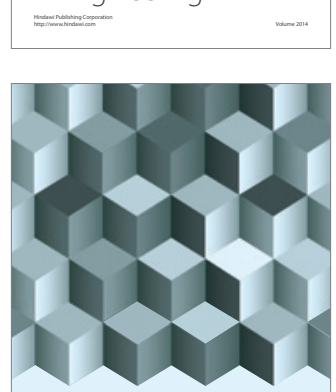

Journal of

Function Spaces
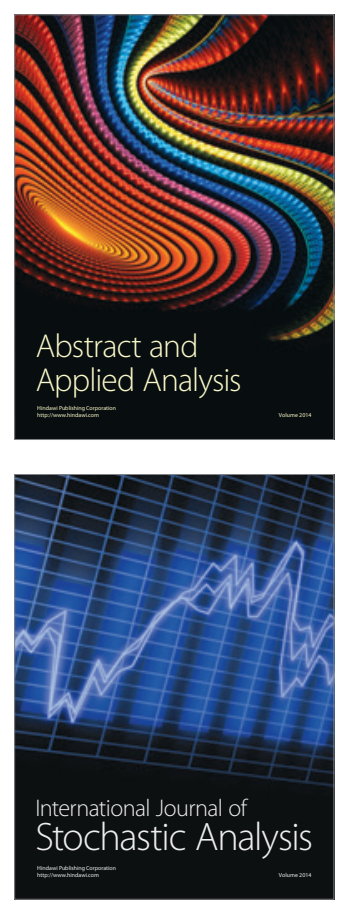

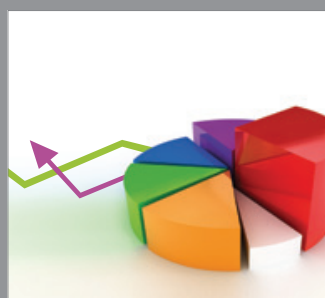

ournal of

Probability and Statistics

Promensencen
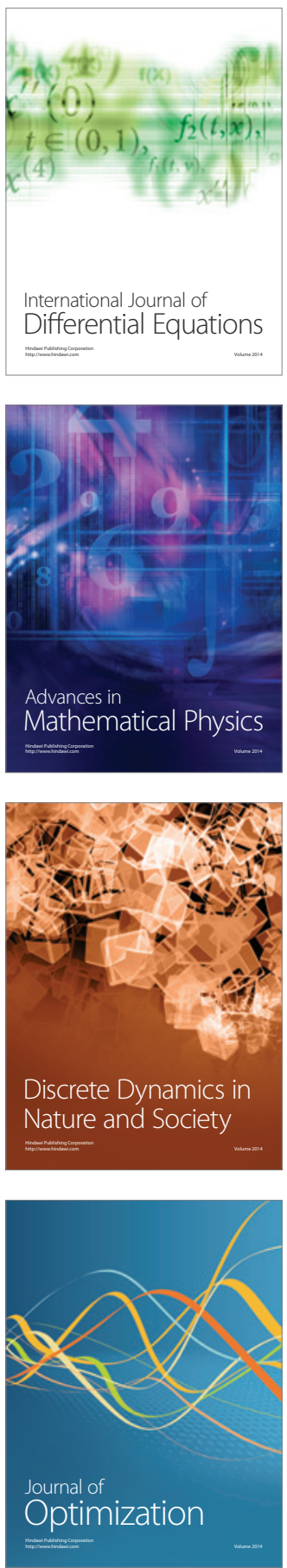